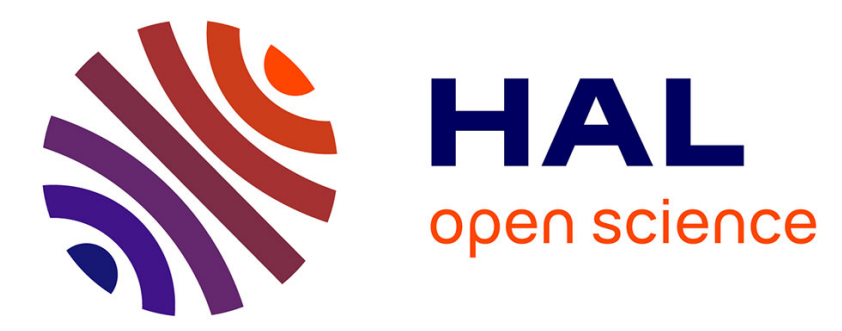

\title{
cAMP-Dependent Co-stabilization of Axonal Arbors from Adjacent Developing Neurons
}

Alice Louail, Martijn Sierksma, Antoine Chaffiol, Sarah Baudet, Ahlem

Assali, Sandrine Couvet, Melissa Nedjam, Fiona Roche, Yvrick Zagar, Jens Duebel, et al.

\section{To cite this version:}

Alice Louail, Martijn Sierksma, Antoine Chaffiol, Sarah Baudet, Ahlem Assali, et al.. cAMPDependent Co-stabilization of Axonal Arbors from Adjacent Developing Neurons. Cell Reports, 2020, 33 (1), pp.108220. 10.1016/j.celrep.2020.108220 . hal-02992307

\section{HAL Id: hal-02992307 https://hal.science/hal-02992307}

Submitted on 9 Nov 2020

HAL is a multi-disciplinary open access archive for the deposit and dissemination of scientific research documents, whether they are published or not. The documents may come from teaching and research institutions in France or abroad, or from public or private research centers.
L'archive ouverte pluridisciplinaire HAL, est destinée au dépôt et à la diffusion de documents scientifiques de niveau recherche, publiés ou non, émanant des établissements d'enseignement et de recherche français ou étrangers, des laboratoires publics ou privés. 


\section{Cell Reports}

\section{cAMP-Dependent Co-stabilization of Axonal Arbors from Adjacent Developing Neurons}

\section{Graphical Abstract}

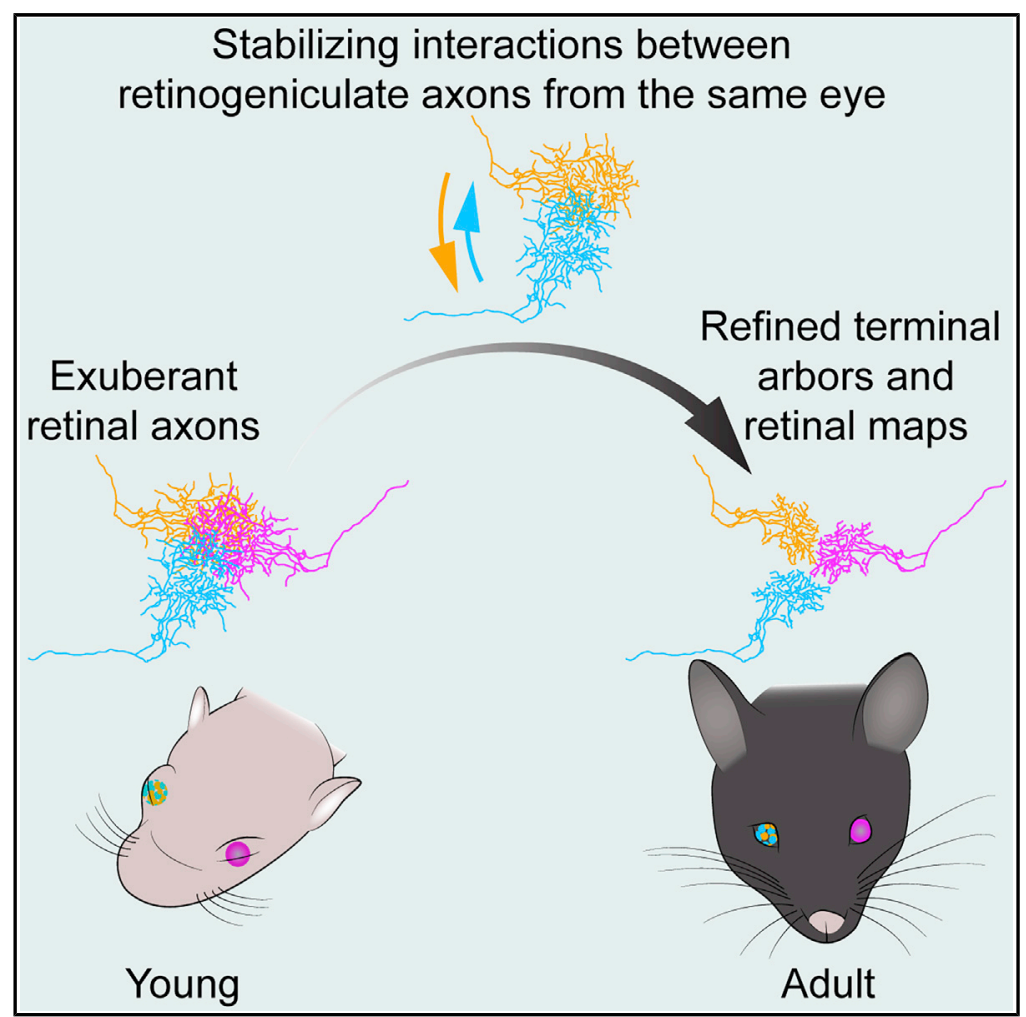

\section{Highlights}

- Neighboring retinal ganglion cells cooperate to co-stabilize their axonal arbors

- Axonal arbor co-stabilization involves cAMP signaling

- cAMP independently controls axon co-stabilization and spontaneous activity

\section{Authors}

Alice Louail, Martijn C. Sierksma, Antoine Chaffiol, ..., Yvrick Zagar, Jens Duebel, Xavier Nicol

\section{Correspondence}

alice.louail@inserm.fr (A.L.), xavier.nicol@inserm.fr (X.N.)

\section{In Brief}

The pruning of axonal arbors is critical to refine early exuberant neuronal connections. Theoretical work suggests that co-active axons co-stabilize their connections. Louail et al. provide experimental evidence confirming this predicted cooperation and identify cAMP signaling as a critical pathway for this developmental process. 


\title{
Report \\ cAMP-Dependent Co-stabilization of Axonal Arbors from Adjacent Developing Neurons
}

\author{
Alice Louail, ${ }^{1,5, *}$ Martijn C. Sierksma, ${ }^{1,3,5}$ Antoine Chaffiol, ${ }^{1}$ Sarah Baudet, ${ }^{1}$ Ahlem Assali, ${ }^{2,4}$ Sandrine Couvet, ${ }^{1}$ \\ Melissa Nedjam, ${ }^{1}$ Fiona Roche, ${ }^{1}$ Yvrick Zagar, ${ }^{1}$ Jens Duebel, ${ }^{1}$ and Xavier Nicol ${ }^{1,6, *}$ \\ ${ }^{1}$ Sorbonne Université, Inserm, CNRS, Institut de la Vision, 17 rue Moreau, Paris 75012, France \\ 2Sorbonne Université, Inserm, UMR_S 1270, Institut du Fer à Moulin, Paris 75005, France \\ ${ }^{3}$ Present address: Department of Neuroscience, Erasmus MC, University Medical Centre Rotterdam, P.O. Box 2040, 3000 CA, Rotterdam, the \\ Netherlands \\ 4Present address: Department of Neuroscience, Medical University of South Carolina, 173 Ashley Avenue, Charleston, SC 29425, USA \\ 5These authors contributed equally \\ 6Lead Contact \\ ${ }^{*}$ Correspondence: alice.louail@inserm.fr (A.L.), xavier.nicol@inserm.fr (X.N.) \\ https://doi.org/10.1016/j.celrep.2020.108220
}

\section{SUMMARY}

Axonal arbors in many neuronal networks are exuberant early during development and become refined by activity-dependent competitive mechanisms. Theoretical work proposed non-competitive interactions between co-active axons to co-stabilize their connections, but the demonstration of such interactions is lacking. Here, we provide experimental evidence that reducing cyclic AMP (cAMP) signaling in a subset of retinal ganglion cells favors the elimination of thalamic projections from neighboring neurons, pointing to a cAMP-dependent interaction that promotes axon stabilization.

\section{INTRODUCTION}

Competitive mechanisms involving synapse stabilization and pruning underlie the development of neuronal connectivity in the nervous system. In amphibians and frogs, they control branch formation and elimination by an electrical activity-dependent pathway and contribute to the developmental refinement of neuronal networks (Ben Fredj et al., 2010; Hua et al., 2005; Ruthazer et al., 2003). Because spontaneous activity of nearby developing neurons is synchronized in many regions of the brain, their co-activity is thought to stabilize their synaptic contacts and to oppose competitive mechanisms (Arroyo and Feller, 2016). This hypothesis has shaped the current model of neuronal circuit development. However, whether axons of co-active neurons influence each other during the refinement of their terminal arbor in physiological conditions lacks experimental confirmation.

Retinofugal projections are an exemplary model to investigate competitive mechanisms shaping neuronal connectivity. In mammals, axonal arbors of developing retinal ganglion cells (RGCs) from both eyes first invade overlapping areas in the dorso-lateral geniculate nucleus (dLGN) before segregating in distinct territories (Godement et al., 1984; Shatz, 1983). The refinement of binocular maps requires competitive mechanisms relying on spontaneous waves of calcium elevation and electrical activity that propagate across the developing retina (Stellwagen and Shatz, 2002). Forcing the synchronization of electrical activity in both retinas reduces the pruning of RGC axons, supporting the hypothesis of competition between asynchronous neurons and/or stabilizing interactions between co-active neurons (Munz et al., 2014; Zhang et al., 2011). The correlation of activity between neighboring RGCs has also been included as a critical component of retinal map development in computational simulations (Godfrey and Swindale, 2014). This suggests that interactions between axons from adjacent RGCs in the retina contribute to the regulation of axon pruning.

The frequency of retinal waves is controlled by cyclic AMP (cAMP) signaling and shapes binocular maps (Penn et al., 1998; Stellwagen and Shatz, 2002). However, the impact of this second messenger is not restricted to the regulation of retinal waves because knocking out adenylyl cyclase 1, a cAMP synthesizing enzyme, prevents the segregation of ipsilateral and contralateral territories (Nicol et al., 2006; Ravary et al., 2003) without altering spontaneous activity in the retina (Dhande et al., 2012; Dunn et al., 2009). The role of a cAMP pathway that does not impact the generation of retinal waves has not been elucidated.

Here, we provide direct evidence that RGCs from the same eye influence each other during the stabilization of their axonal branches via a cAMP-dependent mechanism that does not involve cell-specific changes in the spontaneous activity of the developing retina.

\section{RESULTS}

A molecular approach termed Zaic (short for mosaic) was designed to alter CAMP signaling in a sparse population of RGCs and monitor the axonal development of their neighbors with intact CAMP signaling. Previous sparse labeling approaches in the retina did not enable to trace axons from neurons in the vicinity of labeled or manipulated cells (Badea et al., 2003; Dhande 
A

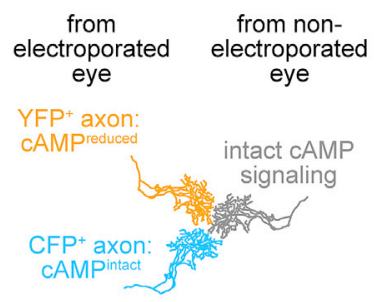

B

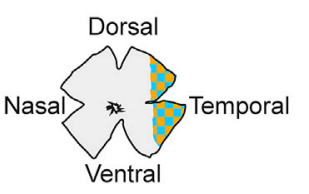

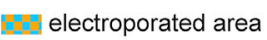

C

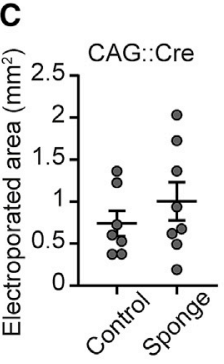

D
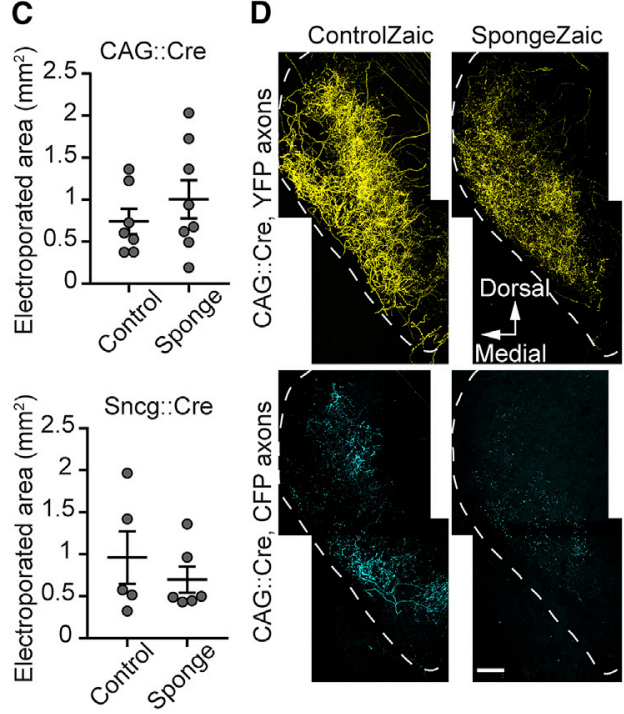

G

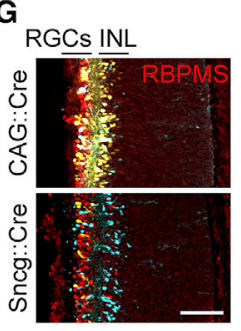

H

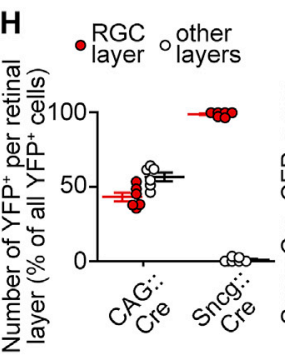

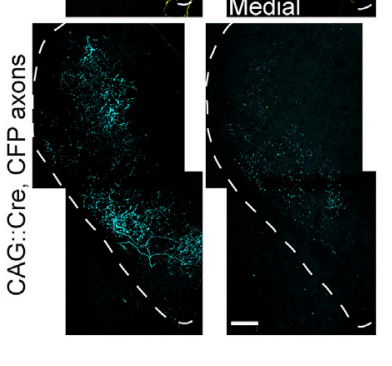

I
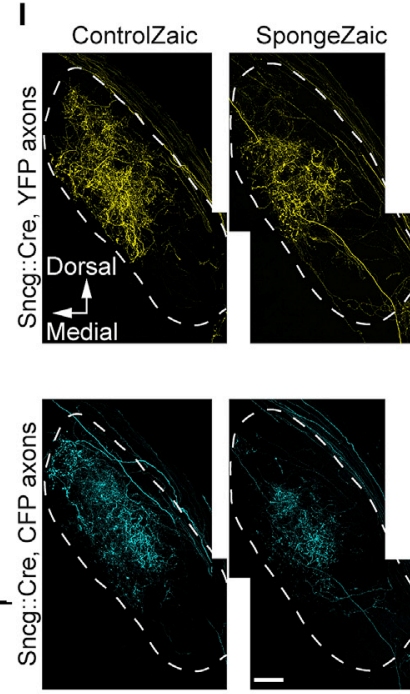

$\mathbf{F}$
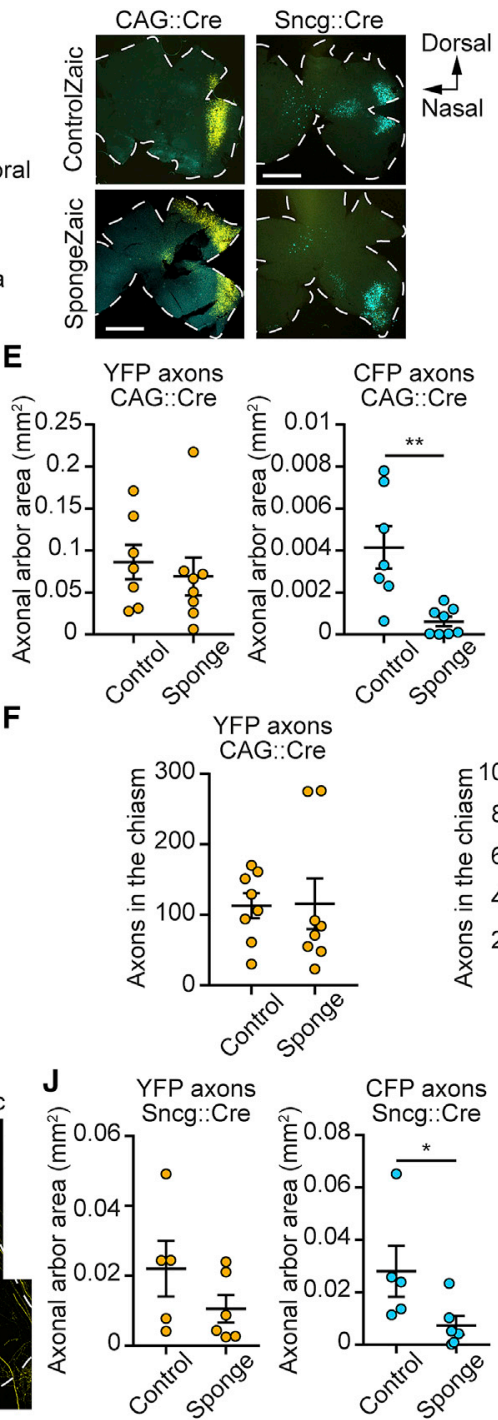

Sncg::Cre
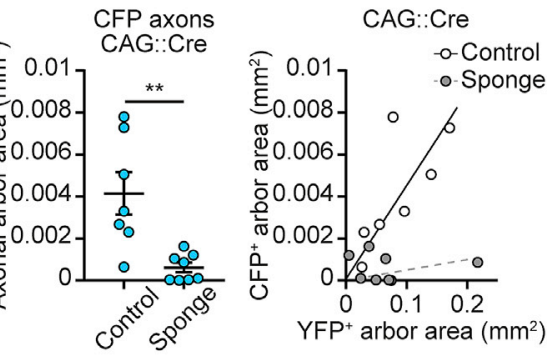

$\mathrm{YFP}^{+}$arbor area $\left(\mathrm{mm}^{2}\right)$

CFP axons

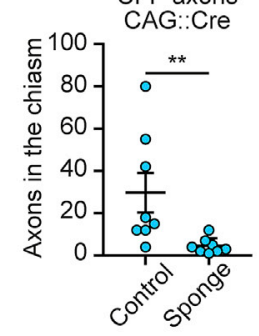

$$
\begin{aligned}
& \text { CFP axons } \\
& \text { Sncg::Cre }
\end{aligned}
$$

Sncg::Cre
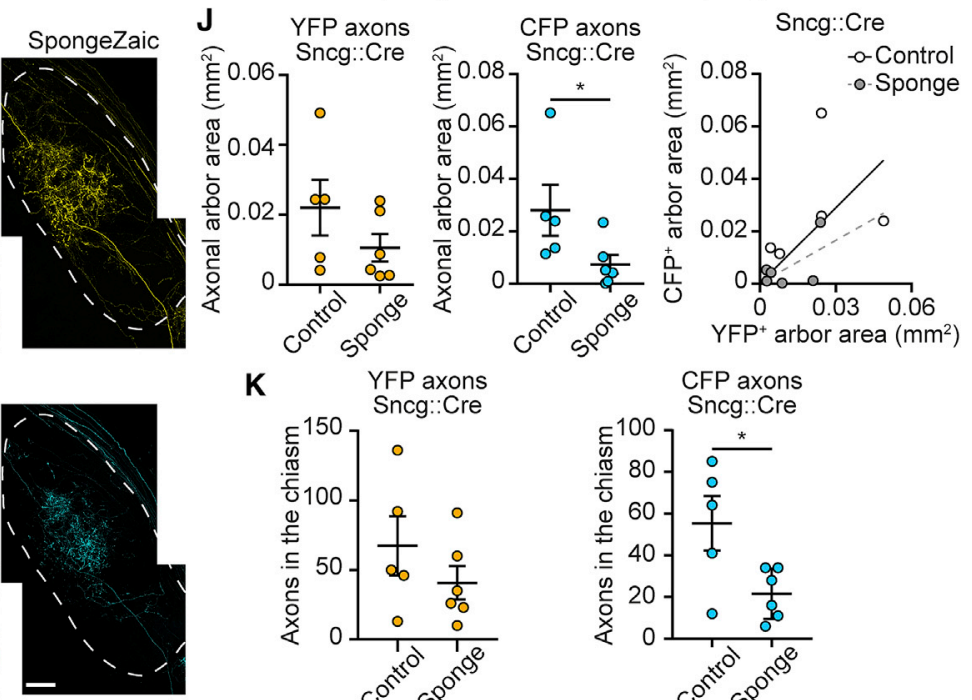

$$
\mathbf{K}
$$

$\mathbf{K}$

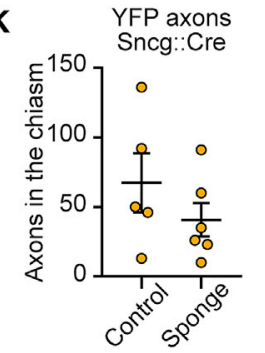

CFP axons

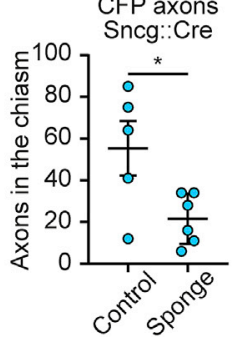

Figure 1. cAMP-Dependent Interactions between Neighboring RGCs during Axon Refinement

(A) The Zaic strategy leads to a mosaic of YFP-expressing neurons with reduced cAMP signaling (cAMPreduced) and CFP-positive neurons with intact signaling (CAMP ${ }^{\text {intact }}$ ) in the retina. This enables the tracing of RGC axons in the dLGN where they are intermingled with unlabeled axons from the same and the opposite eye.

(B) In utero electroporation of the ventro-temporal retina was used to implement the Zaic strategy with Cre expression driven either by the generic CAG promoter or by the RGC-specific Sncg promoter. Drawing shows the orientation of an isolated retina with the desired electroporation area indicated in blue-yellow checkered pattern (left). Dashed lines delineate the contour of the retina (right).

(C) The electroporated area in the retina is similar in ControlZaic and SpongeZaic conditions regardless of the promoter used.

(D) YFP-and CFP-expressing axonal arbors in the contralateral dLGN of either ControlZaic or SpongeZaic electroporated P15 animals (using the CAG promoter). Dashed lines delineate the ventro-medial contour of the dLGN. 
et al., 2011). In contrast, Zaic enables the generation and tracing of two mutually exclusive but intermingled populations of cells. cAMP signaling is altered in one of these cellular populations by using Lyn-cAMP sponge, a genetically encoded cAMP scavenger restricted to lipid rafts that buffers CAMP in cell lines and RGCs (Averaimo et al., 2016; Lefkimmiatis et al., 2009). cAMP sponge contains the cAMP binding sites from the regulatory subunit $1 \beta$ of protein kinase $A$ and acts as a dominant negative for cAMP signaling (Lefkimmiatis et al., 2009). The Zaic approach relies on a DNA construct that contains the CFP sequence (ending with a Stop codon) flanked by two LoxP sites, and followed by Lyn-cAMP sponge fused to YFP by a self-cleaving $2 \mathrm{~A}$ motif (SpongeZaic) (Figure S1A). Co-electroporating SpongeZaic with a low concentration of DNA encoding the Cre recombinase leads to two cellular populations expressing either CFP, with unaffected cAMP signals (cAMP intact), or YFP together with Lyn-cAMP Sponge (CAMPreduced). A similar construct lacking Lyn-cAMP Sponge (ControlZaic) serves as a control. The Zaic strategy was first tested in vitro in HEK293 cells and retinal explants from E14.5 mouse embryos. Lyn-cAMP Sponge expression was detected in Cre and SpongeZaic co-expressing cells, in contrast to cells lacking Cre expression, demonstrating that Cre recombinase is required to drive Lyn-cAMP sponge expression (Figures S1B-S1F). The Zaic:Cre DNA ratio was optimized in vitro using retinal explants (Figures S1E and S1F). $2 \mu \mathrm{g} \times \mu \mathrm{L}^{-1}$ of the Zaic plasmids and $0.1 \mu \mathrm{g} \times \mu \mathrm{L}^{-1}$ of Cre were used in the other experiments. To verify that cAMP signaling is affected in YFP-expressing retinal axons electroporated with SpongeZaic, the SpongeZaic or ControlZaic strategies was implemented in retinal explants expressing R-FlincA, a red fluorescent sensor for CAMP (Ohta et al., 2018). Retinal axons were exposed to a 60 -s pulse of $10 \mathrm{nM}$ forskolin, an activator of transmembrane adenylyl cyclases, resulting in an elevation of CAMP concentration observed by an increase in R-FlincA fluorescence. The amplitude of this elevation was inversely correlated with YFP intensity in SpongeZaic axons, whereas the level of YFP did not influence R-FlincA fluorescence in ControlZaic-electroporated axons, demonstrating that YFP intensity can serve as a proxy for the reduction of cAMP signaling in SpongeZaic RGC axons (Figures S1G-S1I).

We predicted that the need for interactions between neighboring RGCs would be strongest when they are in competition with axons from the other eye. To investigate the impact of cAMP manipulation in a few RGCs on the axonal arbor of their neighbors, SpongeZaic was electroporated in utero in the ventro-temporal embryonic retina of a single eye (Figures $1 \mathrm{~A}$ and 1B). RGCs in this area of the retina project within the contralateral
dLGN to regions close to the territory of the opposite eye. Electroporated axons were traced from the retina and the optic chiasm to the dLGN. The area covered by CFP- and by YFPpositive projections in the dLGN was analyzed at post-natal day $(P) 15$ after eye-specific segregation of axonal arbors using a semi-supervised machine learning-based segmentation method (Figures S1J and S1K). In the contralateral dLGN, both CFP- and YFP-positive axons formed a dense termination zone in ControlZaic animals (Figure 1D). The area covered by cAMP ${ }^{\text {intact }}$ axons in the dLGN (Figures $1 \mathrm{D}$ and $1 \mathrm{E}$ ) and the number of CAMP $^{\text {intact }}$ axons in the chiasm (Figure 1F) were reduced in SpongeZaic animals. This reduction does not reflect variations in the size of the electroporation in the retina (Figures 1B and 1C). In contrast, no significant change in the area covered by cAMPreduced axons was found (Figures 1D and 1E). cAMPreduced axons might differ from their cAMP ${ }^{\text {intact }}$ neighbors because of their cell-autonomous reduced ability to retract, previously identified in the context of ephrinA-induced axon guidance in the superior colliculus (Averaimo et al., 2016). To evaluate whether our observations are affected by the extent of the electroporation, we plotted the area covered by CFPexpressing axons against the area of the YFP-positive arborizations for individual animals and fitted the results to a straight line. The slope of the fit is reduced in the SpongeZaic-electroporated animals compared to ControlZaic brains (Figure 1E), confirming the specific reduction in the area covered by cAMPintact axons. The results demonstrate that reducing cAMP signaling in a restricted number of retinal neurons (cAMPreduced) affects the development of neighboring RGCs in which cAMP signals were not manipulated (cAMPintact).

CAMP signaling in amacrine cells is essential for the modulation of patterned spontaneous activity in the retina (Hsiao et al., 2019) and for the refinement of retinal projections (Nicol et al., 2006; Ravary et al., 2003). Because adenylyl cyclase 1 is not expressed in amacrine cells and is critical for binocular maps to refine (Nicol et al., 2006; Ravary et al., 2003), cAMP signaling in RGCs must also be involved. To determine the identity of the retinal cells requiring CAMP signaling for the stabilization of CAMP $^{\text {intact }}$ axon branches, the non-specific CAG promoter used to drive Cre expression was replaced by the RGC-specific $\gamma$-synuclein (Sncg) promoter (Chaffiol et al., 2017). The control of Cre expression by the Sncg promoter led to the restriction of YFP expression to the RGC layer, immunostained by the RGC marker RNA-binding protein with multiple splicing (RBPMS) (Figures $1 \mathrm{G}$ and $1 \mathrm{H}$ ). The area covered by cAMP $^{\text {intact }}$ axons in the dLGN and the number of axons in the chiasm were reduced at P15 in SpongeZaic-electroporated

(E) The extent of the cAMP ${ }^{\text {intact }}$ axonal arbors (CFP-positive) is reduced in SpongeZaic-electroporated animals. Plotting the area covered by cAMP intact arbors

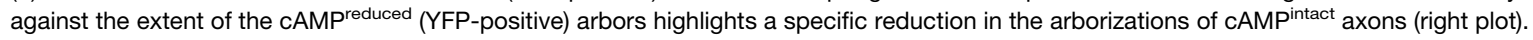

(F) The number of CFP-positive axons detected in the optic chiasm is diminished in SpongeZaic-electroporated animals.

(G) Driving expression of the Cre recombinase by the RGC-specific Sncg promoter rather than the non-specific CAG promoter leads to the restriction of YFP to the RGC layer. RGCs are labeled using a RBPMS antibody (red) at P3.

$(\mathrm{H})$ When Sncg::Cre is used, YFP-positive cells are found almost exclusively in the RGC layer but not in the inner nuclear layer (INL).

(I-K) Reducing cAMP signaling in a sparse population of RGCs using the Sncg promoter leads to a reduction (J) in the area covered by cAMPintact axons (CFPpositive) in the dLGN at $\mathrm{P} 15$ and $(\mathrm{K})$ in the number of CAMP ${ }^{\text {intact }}$ axons in the optic chiasm.

Scale bars, $1 \mathrm{~mm}(\mathrm{~B}) ; 50 \mu \mathrm{m}(\mathrm{D}, \mathrm{G}$, and $\mathrm{I})$. Data are mean \pm SEM. (C, E, F, J, and K) Values for individual animals are shown. (H) Values for individual sections are shown. ${ }^{*} \mathrm{p}<0.05 ;{ }^{\star *} \mathrm{p}<0.01$; Mann-Whitney. Exact $\mathrm{p}$ values and number of replicates in Table S1.

See also Figures S1, S2, and S4. 
A

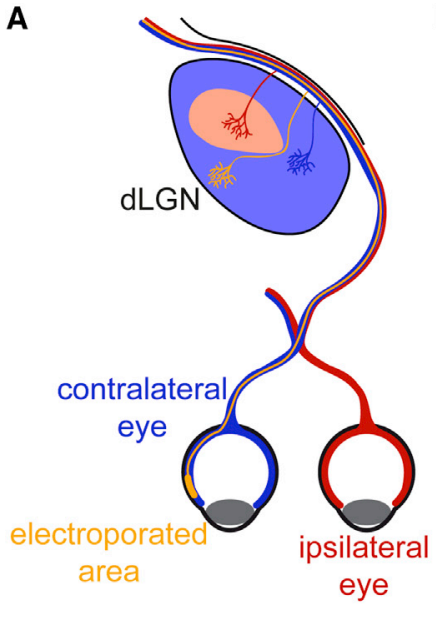

B

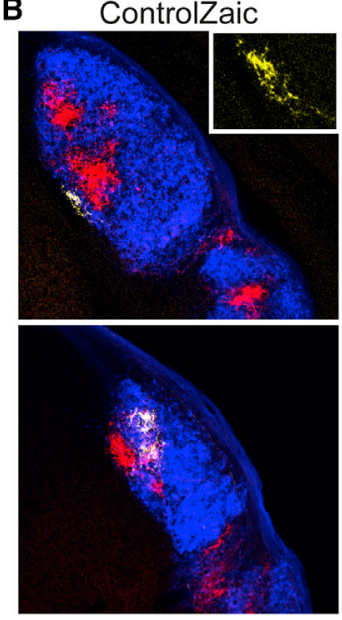

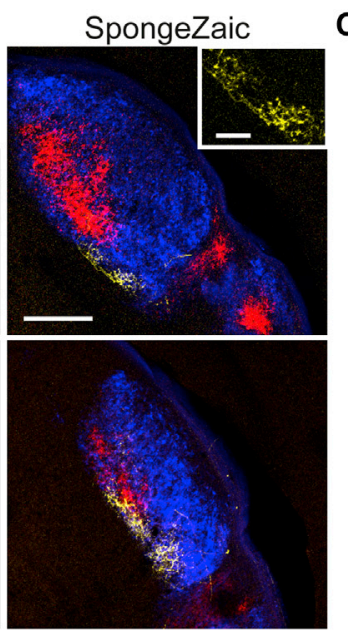

C

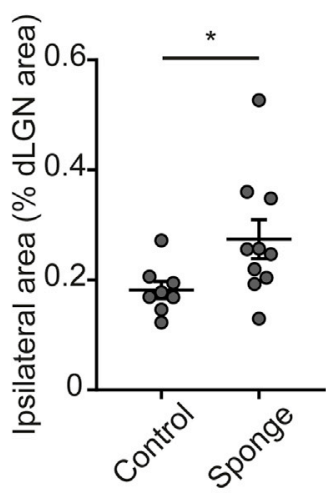

Figure 2. Altering cAMP Signaling in Sparse RGCs Affects the Overall Development of Binocular Maps

(A) Lyn-CAMP sponge was expressed in sparse RGCs from the ventro-temporal crescent of the developing retina (yellow) using in utero electroporation. The same animal was injected into the eyes at P13 with Alexa647- (electroporated eye, blue) or Alexa594- (non-electroporated eye, red) cholera toxin subunit $\beta$. The dLGN contralateral to the electroporated eye was imaged.

(B and C) The ipsilateral territory (red, from the non-electroporated retina) is enlarged in Lyn-cAMP Sponge-expressing animals at P15. The electroporated YFPpositive axons (inset) are found in the contralateral territory. Two sections of the dLGN from the same animal are shown.

Scale bars, $200 \mu \mathrm{m}$ (A); $50 \mu \mathrm{m}$ (inset). (B) Data are mean \pm SEM. Values for individual animals are shown. ${ }^{*} p<0.05$; Mann-Whitney. Exact $p$ values and number of replicates in Table $\mathrm{S} 1$.

animals compared to their controls (Figures 1I-1K). Like in CAG::Cre-electroporated mice, no significant change in the area covered by CAMPreduced axons was found (Figures 11 and $1 \mathrm{~J})$. Overall, reducing cAMP signaling only in $\mathrm{RGCs}$ reproduces the phenotype observed when Lyn-cAMP sponge is expressed throughout the retinal layers, demonstrating that the stabilization of retinal axon branches requires CAMP signaling in neighboring RGCs. Of note, using the Sncg instead of the CAG promoter also reduces the ratio between the area covered by CFP and YFP axons in the dLGN of ControlZaic-electroporated animals ( 1:20 in CAG::Cre animals, $\sim 1: 1$ in Sncg::Cre animals) (Figures $1 \mathrm{D}, 1 \mathrm{E}, 1 \mathrm{I}$, and $1 \mathrm{~J})$, without preventing the reduction in CAMP ${ }^{\text {intact }}$ axonal branches in SpongeZaic-electroporated animals. Combined with the observation that in the electroporated area only few RGCs express either CFP or YFP (Figure 1G), this suggests that reducing CAMP signaling in a minority of RGCs is sufficient to alter the projections of neighboring CAMP $^{\text {intact }}$ axons.

To evaluate whether the influence of cAMP signaling in CAM$P^{\text {reduced }}$ axons on their cAMP ${ }^{\text {intact }}$ neighbors is restricted to the ventro-temporal end of the retina, we reproduced similar experiments with electroporation performed in the central part of the retina. Like when the electroporation was performed in the ventro-temporal retina, the area covered by cAMP ${ }^{\text {intact }}$ axons was reduced in SpongeZaic-electroporated animals compared to ControlZaic-electroporated animals, generalizing our findings to the entire retina (Figure S2).

To investigate whether altering cAMP signaling in a few retinal neurons impacts the projections from the non-electroporated retina, the Zaic strategy conducted in the ventro-temporal retina was combined with tracing the complete projections of each eye using fluorescent cholera toxins (Figure 2A). The area covered by the ipsilateral projections of the non-electroporated eye was enlarged in Lyn-cAMP sponge-expressing animals (Figures 2B and $2 \mathrm{C}$ ), demonstrating that altering CAMP signaling in a sparse number of neurons of one retina is sufficient to influence binocular competition.

The reduction in the terminal arbor size and number of cAMP $^{\text {intact }}$ axons in SpongeZaic-electroporated animals might reflect abnormalities in early development of retinal projections prior to their pruning. To identify the affected developmental stage, embryonic day (E) 14.5 embryos were electroporated using the Zaic strategy and pups were harvested at P3, after retinal axons have reached the dLGN, but before eye-specific map refinement (Godement et al., 1984; Huberman et al., 2008). SpongeZaic-electroporated axons invaded the dLGN to the same extent as their controls at P3 (Figures 3A-3C) and were as numerous in the chiasm (Figure $3 \mathrm{D}$ ), suggesting a minimal role of errors in axonogenesis, axon outgrowth, or axon pathfinding. Therefore, the reduction of CAMP $^{\text {intact }}$ arbors arises while RGC axons are pruned.

Programmed cell death is involved in the refinement of visual axons (Fawcett et al., 1984) and peaks at P3 (Young, 1984). Because the reduction in the number of CAMP $^{\text {intact }}$ axons in the chiasm occurs between P3 and P15 in SpongeZaic-electroporated animals, we performed TUNEL staining to assess the impact of SpongeZaic on developmental cell death. The proportion of TUNEL-positive cells among cAMPreduced and cAMP ${ }^{\text {intact }}$ RGCs did not differ from RGCs in ControlZaic retinas (Figures 3E-3G), indicating that Lyn-cAMP sponge expression does not alter cell death at this stage.

Reducing spontaneous electrical activity in one retina diminishes the size of the territory occupied by the projections from this eye (Penn et al., 1998). Because cAMP signaling modulates the activity of developing RGCs (Stellwagen et al., 1999), we 
A
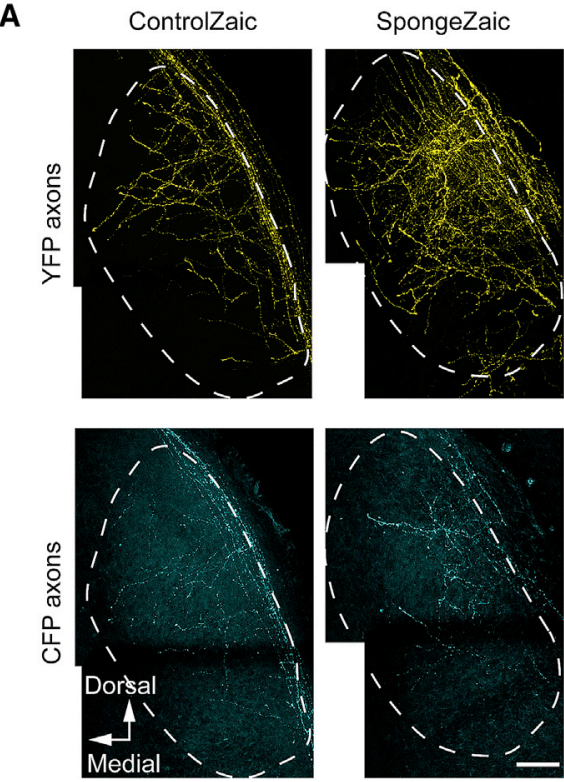

E positive control (DNAse)

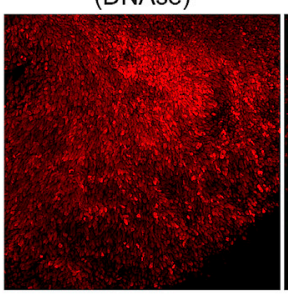

$\mathbf{F}$
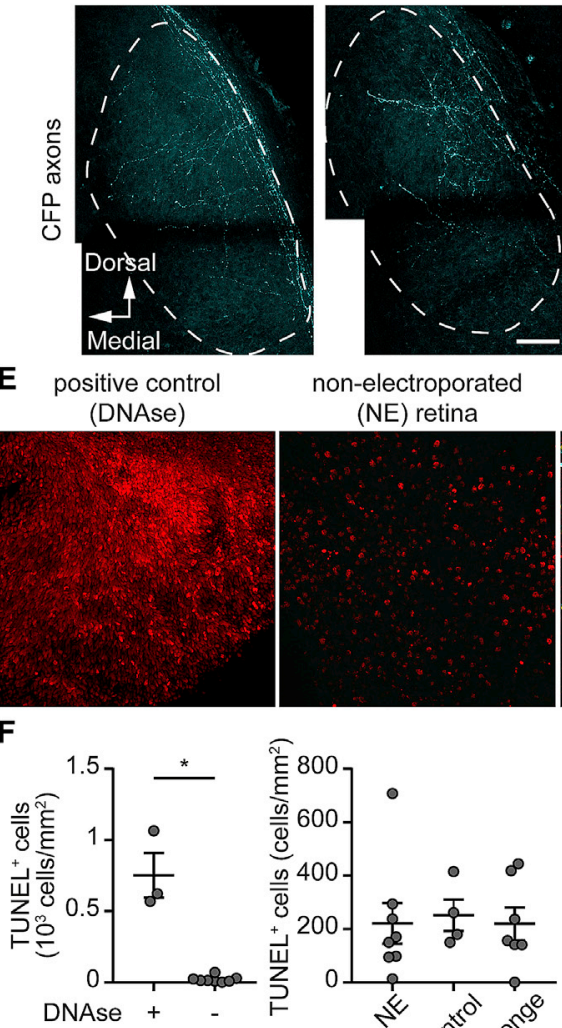

non-electroporated (NE) retina
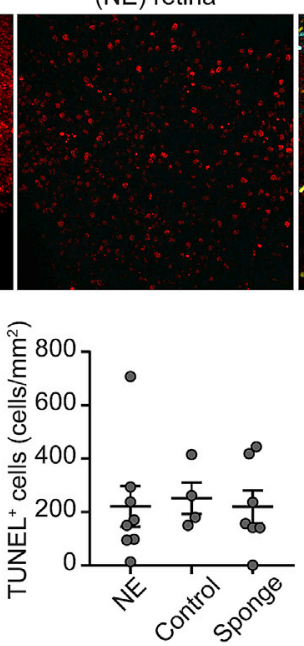

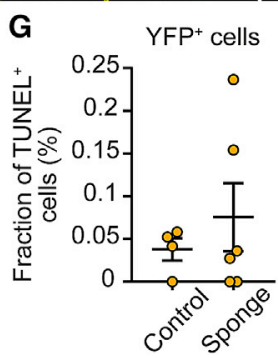

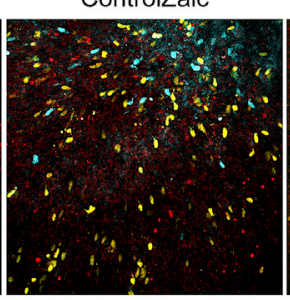

ControlZaic

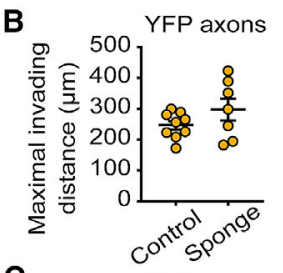

C

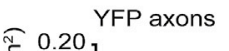

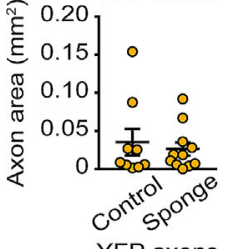

D

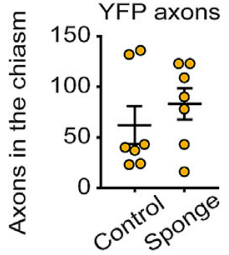

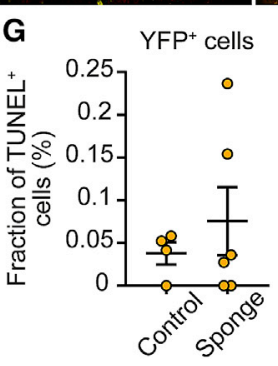

evaluated the impact of Lyn-cAMP sponge using whole-cell electrophysiology. Either the CAG or Sncg promoter was used to drive Lyn-cAMP sponge expression. No difference was observed in RGC resting properties or action potential waveforms (Figures S3A-S3H). When monitoring spontaneous activity, neither the firing frequency, nor the number of action potentials within a burst were different for either CAMP $^{\text {reduced }}$ or CAMP $^{\text {intact }}$ neurons compared to their respective controls (ControlZaic) at P7-8 or P10-11 (Figures S3I-S3L). The burst frequency of CAMPintact neurons might be slightly elevated compared to CFP-positive RGCs in ControlZaic animals at P78 (Sncg::Cre, $p^{\prime}=0.08$ but CAG::Cre, $p^{\prime}=0.34$ ), but this elevation did not persist until $\mathrm{P} 10-11$, and the burst frequency was not different from wild-type retinas (Figures 4 A and S3I-S3L). In the context of competitive interactions, increased activity would lead to an extension of the territory of the over-active RGCs (Penn et al., 1998) and is thus not the reason of the observed

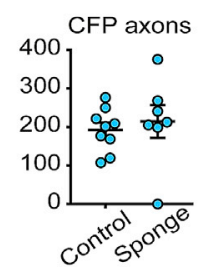

0.003 CFP axons

0.002

0.001 .

01.

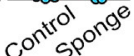

CFP axons

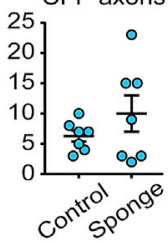

SpongeZaic

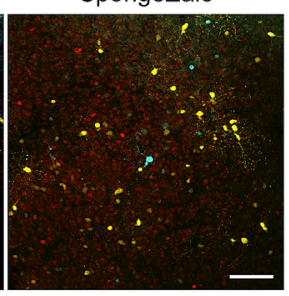

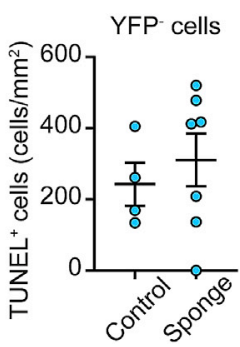

Figure 3. cAMP Signaling Reduction in a Few RGCs Does Not Prevent Retinal Axons from Reaching the dLGN or Alter Developmental Cell Death

(A) CAMP $^{\text {reduced }}$ and $\mathrm{CAMP}^{\text {intact }}$ retinal axons are found at P3 in the dLGN of SpongeZaic-electroporated animals with the same extent as the CFPand YFP-expressing axons respectively after ControlZaic electroporation. Dashed lines delineate the contour of the dLGN.

(B) The longest five axons have a similar length in the dLGN, regardless of their expression of LyncAMP sponge.

(C and D) The area covered by axons (C) and the number of axons in the optic chiasm (D) are similar in SpongeZaic and ControlZaic animals, indicating that early development of retinal axons (including axonogenesis and pathfinding to their targets) is not affected.

(E) Retinal cells undergoing cell death were TUNEL stained in whole mount $\mathrm{P} 3$ retinas.

(F) Treatment of non-electroporated retina with DNase as a positive control leads to a high density of TUNEL-positive cells, in contrast to retinas from either non-electroporated animals, ControlZaic or SpongeZaic-electroporated pups. Lyn-cAMP Sponge expression does not impact the overal number of TUNEL-positive cells.

(G) Neither the proportion of TUNEL-labeled cells among YFP-positive neurons, nor the number of YFP-negative neurons undergoing cell death was affected by Lyn-cAMP Sponge expression.

Scale bars, $50 \mu \mathrm{m}$. Data are mean \pm SEM. * $\mathrm{p}<$ 0.05 ; Mann-Whitney except for in ( $F$; right) Kruskal-Wallis. Values for individual animals are shown. Exact $p$ values and number of replicates in Table S1.

reduction in the CAMP ${ }^{\text {intact }}$ arbor size. Of note, nearby cells did fire more action potentials per burst in all retinas (including controls) leading to an increase in average firing (Figures S3I-S3L). This may reflect a difference in RGC subtype or maturation between electroporated and nearby neurons. It is not related to the expression of Lyn-cAMP Sponge and thus cannot explain the reduction in the extent of $\mathrm{CAMP}^{\text {intact }}$ arbors in SpongeZaic-electroporated animals.

The desynchronization of RGC activity favors axon pruning (Huberman et al., 2008; Stellwagen and Shatz, 2002). To assess the synchronization of RGC activity, we monitored calcium waves in P7-8 retinas (Figures 4B-4I; Video S1). The frequency of retinal waves was not affected when Lyn-cAMP sponge was expressed throughout the retinal layers and diminished with RGC-specific expression of Lyn-cAMP sponge (Figure 4E), excluding the cAMP-dependent regulation of the frequency of retinal waves to be the explanatory basis of the reduction in cAMP ${ }^{\text {intact }}$ axons. The cells in SpongeZaic retinas were more likely to be active during a calcium wave, regardless of their expression of Lyn-cAMP sponge (Figures $4 \mathrm{D}$ and $4 \mathrm{~F}-4 \mathrm{I}$; Video S1). Although the reduction of CAMPintact 
A

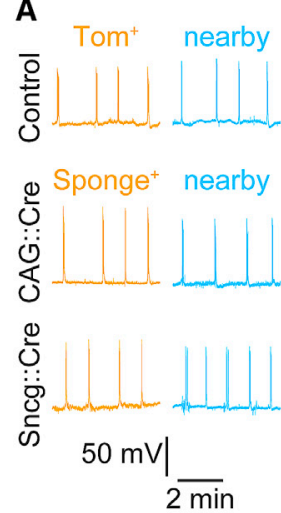

D

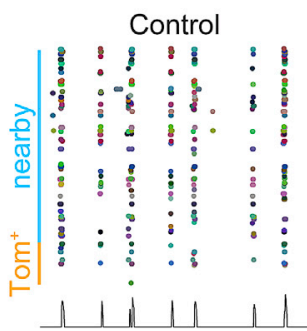

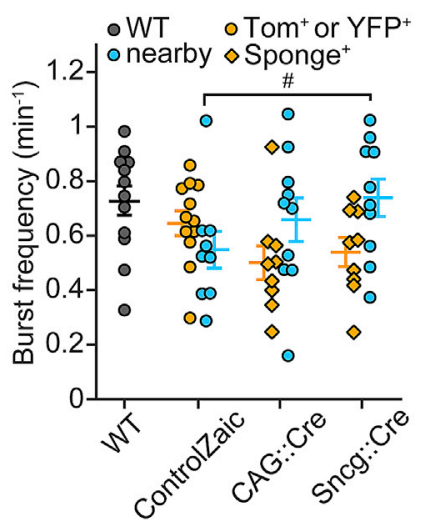

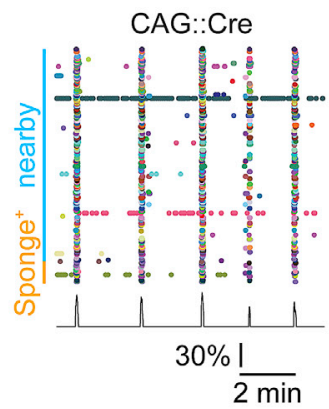

E
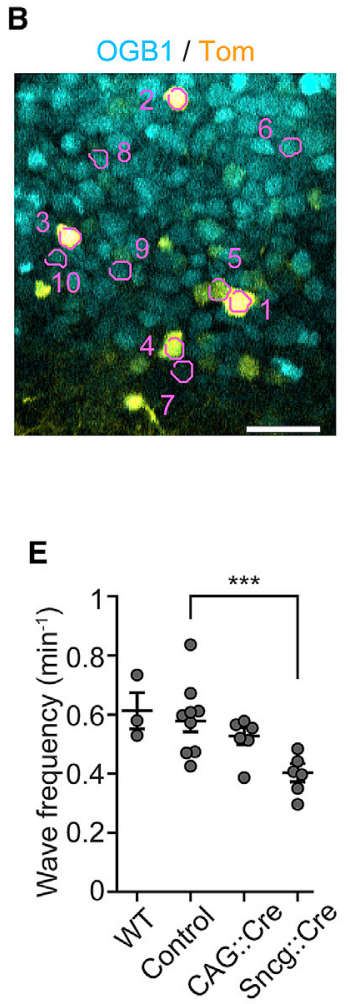

C

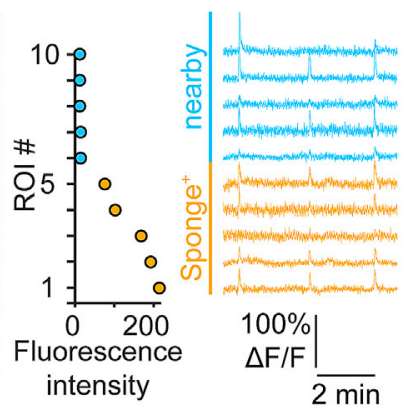

F $\quad$ oWT $\diamond$ Sponge $^{+}$ ० Tom ${ }^{+}$o nearby

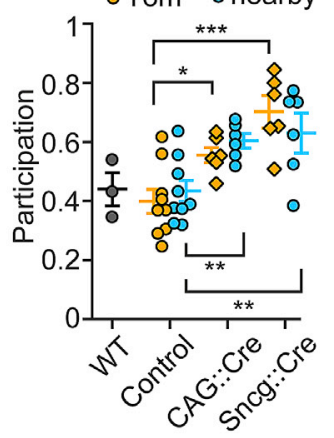

G
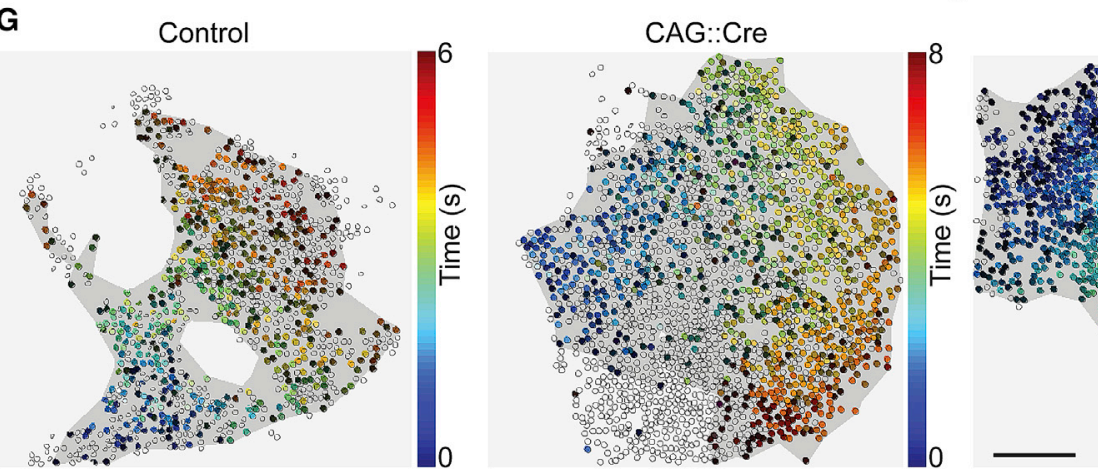

Sncg::Cre

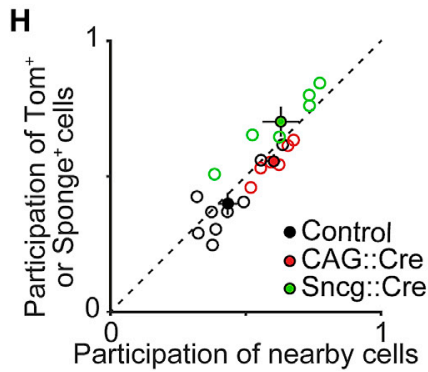

I

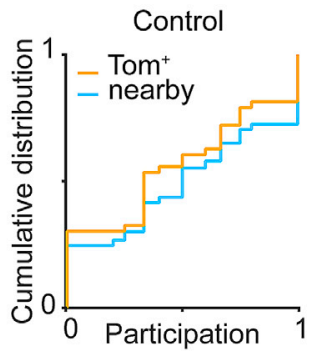

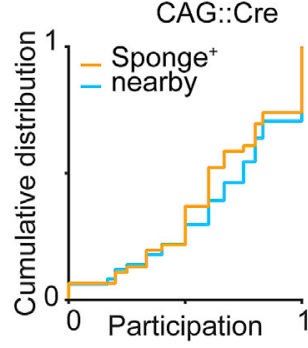

Shco:.

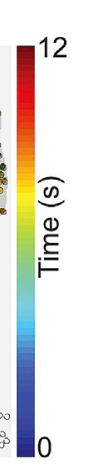

12

\section{.}


axon arbors and the alteration in eye-specific territories in SpongeZaic-electroporated animals may have been the result of a reduced synchronization (Arroyo and Feller, 2016), we observed an increase in synchronization. We therefore consider that the mechanism targeted by Lyn-cAMP sponge leading to the reduction in CAMP $^{\text {intact }}$ axon arbors is not a consequence of changes in retinal activity.

\section{DISCUSSION}

Overall, we demonstrated that RGCs influence the stabilization of the axonal branches of their neighbors during axon pruning via a RGC-specific, cAMP-dependent signaling pathway. This suggests a non-cell-autonomous regulation of axonal arbors via cAMP signaling. This cAMP-dependent interaction, direct or indirect via a postsynaptic neuron or glial cells, is either cooperative or competitive in nature (Arroyo and Feller, 2016). In the framework of a non-cell-autonomous regulation relying on CAMP, altering the signaling of this cellular messenger can preclude RGCs either to emit a signal that influences their neighbors or to be sensitive to a cue from the neighboring neurons. This yields four possibilities, each with specific experimental predictions (Figure S4). If CAMP is required in the $R G C$ that receives a (1) competitive (i.e., destabilizing) or (2) co-stabilizing signal from its neighbors, one would anticipate the terminal arbor of CAMP $^{\text {reduced }}$ axons to be changed without affecting CAMP ${ }^{\text {intact }}$ axons. (3) If CAMP is required in the $R G C$ initiating a competitive (i.e., destabilizing) signal for its neighbors, CAMPintact arbors are expected to expand when CAMP is altered in CAMPreduced axons. None of these possibilities is reflected in our results. In contrast, (4) if cAMP signaling is required in the RGC sending a co-stabilizing signal, one would expect a reduction of the CAMP ${ }^{\text {intact }}$ arbors, as observed in our experiments. We thus favor the explanation that CAMP signaling is required for the generation of a signal emitted by RGC axons that stabilizes the branches of their neighboring neurons in the retina.

One might have anticipated the projections of $\mathrm{CAMP}^{\text {reduced }}$ neurons to be reduced to a similar extent to the CAMP ${ }^{\text {intact }}$ axons. The difference between these two populations might rely on the altered ability to retract of axons expressing Lyn-cAMP Sponge. This cell-autonomous mechanism, that thus cannot affect cAMP $^{\text {intact }}$ axons, has been identified in the context of ephrinA- induced axon repulsion (Averaimo et al., 2016). Because axon pruning coincides with waves of electrical activity and the expression of adenylyl cyclase 1 in many sensory systems during development (Nicol and Gaspar, 2014; Yamamoto and López-Bendito, 2012), we anticipate that the cAMP-dependent interactions between presynaptic neurons is key for the development of the nervous system.

\section{STAR $\star$ METHODS}

Detailed methods are provided in the online version of this paper and include the following:

- KEY RESOURCES TABLE

- RESOURCE AVAILABILITY

O Lead Contact

O Materials Availability

O Data and Code Availability

- EXPERIMENTAL MODEL AND SUBJECT DETAILS

O Animals

O Cell lines

O Retinal explants

- METHOD DETAILS

O Plasmids

- WESTERN-BLOT ANALYSIS

O HEK293 cell transfection

O In utero retinal electroporation

○ Confocal imaging

O Plasmids electroporated

$\bigcirc$ Retina slicing and immunostaining

$\checkmark$ Two-photon calcium imaging

O Whole-cell electrophysiology

$\bigcirc$ TUNEL staining and analysis

O R-FlincA imaging

Anterograde tracing

- QUANTIFICATION AND STATISTICAL ANALYSIS

Axon area analysis

$O$ Analysis of the distribution of ipsilateral and contralateral fibers in the dLGN

O Analysis of maximal invading distance

C Calcium imaging analysis

O Defining ROls

Activity of the ROls

(D) Activity of $300 \mathrm{ROI}$ (top, raster plot with every line showing activity of a color-coded ROI) and participation in retinal waves (bottom).

(E) The frequency of retinal waves is reduced when Lyn-cAMP Sponge expression is driven by Sncg::Cre but not CAG::Cre.

(F) The participation rate of both cAMP ${ }^{\text {intact }}$ and cAMPreduced neurons is increased.

(G) Example of spontaneous calcium waves from a control retina (left), from a retina expressing Lyn-cAMP Sponge without cell-type specificity (CAG::Cre, middle), and from a retina expressing Lyn-cAMP sponge in a few RGCs (Sncg::Cre, right). The field of view is shown for each retina with the time points of activity of ROls coded in color. The darker gray area shows the area covered by this wave within the field of view. Open contours indicate ROI that were not active in this wave. Due to the curvature in the retina some parts of the RGC layer are out of focus.

(H) Participation of ROI with expression of tdTomato alone (Control) or together with Lyn-cAMP sponge versus participation from the nearby cells without expression of tdTomato and Lyn-cAMP sponge. Open circle represents an individual retina, closed circle represents the average, and the SEM is also shown. Dotted line indicates $y=x$.

(I) Cumulative distribution of ROI participation from a control retina (left), from a retina expressing lox-STOP-lox Lyn-cAMP sponge-2A-tdTom together with the CAG::Cre plasmid (middle), and from a retina expressing lox-STOP-lox Lyn-CAMP sponge-2A-tdTom together with the Sncg::Cre plasmid (right).

Scale bar, $25 \mu \mathrm{m}(\mathrm{B}) ; 100 \mu \mathrm{m}(\mathrm{G})$. Data are mean \pm SEM. Values for individual retinas in (E), (F), and (H) or cells in (A) are shown. \# $p^{\prime}<0.10,{ }^{*} p^{\prime}<0.05,{ }^{* *} p^{\prime}<0.01$, ${ }^{* * *} p^{\prime}<0.001$; Conover-Iman. Exact $p$ values and number of replicates in Table S1.

See also Figure S3. 


\section{O Defining waves \\ O Electrophysiology analysis \\ $O$ R-FlincA imaging analysis \\ ○ Statistics}

\section{SUPPLEMENTAL INFORMATION}

Supplemental Information can be found online at https://doi.org/10.1016/j. celrep.2020.108220.

\section{ACKNOWLEDGMENTS}

We thank Dr. Horikawa for the gift of the R-FlincA plasmid. We are grateful to Jean Livet for help in designing the Zaic strategy, to Nicholas Spitzer and Patricia Gaspar for helpful comments, to members of our labs and of J. Livet lab for thoughtful discussions, to Corentin Joffrois and Serge Picaud for access to a patch-clamp electrophysiology setup, and to the members of the animal and imaging facilities of Institut de la Vision. This work was supported by grants from ANR (ANR-15-CE16-0007-01), UNADEV (17UU1166-00), and Sorbonne Université (FCS-SU IDEX SUPER SU-15-R-PERSU-17) to X.N. This work was performed in the framework of the LABEX LIFESENSES (ANR-10LABX-65) and IHU FOReSIGHT (ANR-18-IAHU-0001) supported by French state funds managed by the Agence Nationale de la Recherche within the Investissements d'Avenir program. A.L. was supported by a fellowship from the ED3C doctoral program (Sorbonne Université).

\section{AUTHOR CONTRIBUTIONS}

A.L., M.C.S., and X.N. designed the experiments. A.L., S.C., F.R., and Y.Z. performed molecular biology experiments. A.L. performed axon morphology experiments and analysis. A.A. contributed to axon morphology experiments. F.R. performed ex vivo experiments and analysis. S.B. performed the cAMP imaging experiments using the R-FlincA sensor. M.C.S. performed experiments and analysis for electrophysiology recordings. M.C.S. and A.C. performed and analyzed 2-photon calcium imaging. A.L. and M.N. performed cell death experiments and analysis. A.L. wrote the initial draft. A.L., M.C.S., and X.N. prepared the manuscript.

\section{DECLARATION OF INTERESTS}

The authors declare no competing interests.

Received: January 2, 2020

Revised: July 30, 2020

Accepted: September 11, 2020

Published: October 6, 2020

\section{REFERENCES}

Arganda-Carreras, I., Kaynig, V., Rueden, C., Eliceiri, K.W., Schindelin, J., Cardona, A., and Sebastian Seung, H. (2017). Trainable Weka Segmentation: a machine learning tool for microscopy pixel classification. Bioinformatics 33 , 2424-2426.

Arroyo, D.A., and Feller, M.B. (2016). Spatiotemporal Features of Retinal Waves Instruct the Wiring of the Visual Circuitry. Front. Neural Circuits 10, 54 .

Averaimo, S., Assali, A., Ros, O., Couvet, S., Zagar, Y., Genescu, I., Rebsam, A., and Nicol, X. (2016). A plasma membrane microdomain compartmentalizes ephrin-generated cAMP signals to prune developing retinal axon arbors. Nat. Commun. 7, 12896.

Badea, T.C., Wang, Y., and Nathans, J. (2003). A noninvasive genetic/pharmacologic strategy for visualizing cell morphology and clonal relationships in the mouse. J. Neurosci. 23, 2314-2322.

Ben Fredj, N., Hammond, S., Otsuna, H., Chien, C.-B., Burrone, J., and Meyer, M.P. (2010). Synaptic activity and activity-dependent competition regulates axon arbor maturation, growth arrest, and territory in the retinotectal projection. J. Neurosci. 30, 10939-10951.

Chaffiol, A., Caplette, R., Jaillard, C., Brazhnikova, E., Desrosiers, M., Dubus, E., Duhamel, L., Macé, E., Marre, O., Benoit, P., et al. (2017). A New Promoter Allows Optogenetic Vision Restoration with Enhanced Sensitivity in Macaque Retina. Mol. Ther. 25, 2546-2560.

Dhande, O.S., Hua, E.W., Guh, E., Yeh, J., Bhatt, S., Zhang, Y., Ruthazer, E.S., Feller, M.B., and Crair, M.C. (2011). Development of single retinofugal axon arbors in normal and $\beta 2$ knock-out mice. J. Neurosci. 31, 3384-3399.

Dhande, O.S., Bhatt, S., Anishchenko, A., Elstrott, J., Iwasato, T., Swindell, E.C., Xu, H.-P., Jamrich, M., Itohara, S., Feller, M.B., and Crair, M.C. (2012) Role of adenylate cyclase 1 in retinofugal map development. J. Comp. Neurol. 520, 1562-1583.

Dunn, T.A., Storm, D.R., and Feller, M.B. (2009). Calcium-dependent increases in protein kinase-A activity in mouse retinal ganglion cells are mediated by multiple adenylate cyclases. PLoS ONE 4, e7877.

Fawcett, J.W., O'Leary, D.D., and Cowan, W.M. (1984). Activity and the control of ganglion cell death in the rat retina. Proc. Natl. Acad. Sci. USA 81, 55895593.

Godement, P., Salaün, J., and Imbert, M. (1984). Prenatal and postnatal development of retinogeniculate and retinocollicular projections in the mouse. J. Comp. Neurol. 230, 552-575.

Godfrey, K.B., and Swindale, N.V. (2014). Modeling development in retinal afferents: retinotopy, segregation, and ephrinA/EphA mutants. PLoS ONE 9 e104670.

Hsiao, Y.-T., Shu, W.-C., Chen, P.-C., Yang, H.-J., Chen, H.-Y., Hsu, S.-P., Huang, Y.-T., Yang, C.-C., Chen, Y.-J., Yu, N.-Y., et al. (2019). Presynaptic SNAP-25 regulates retinal waves and retinogeniculate projection via phosphorylation. Proc. Natl. Acad. Sci. USA 116, 3262-3267.

Hua, J.Y., Smear, M.C., Baier, H., and Smith, S.J. (2005). Regulation of axon growth in vivo by activity-based competition. Nature 434, 1022-1026.

Huberman, A.D., Feller, M.B., and Chapman, B. (2008). Mechanisms underlying development of visual maps and receptive fields. Annu. Rev. Neurosci. 31 479-509.

Lefkimmiatis, K., Moyer, M.P., Curci, S., and Hofer, A.M. (2009). "cAMP sponge": a buffer for cyclic adenosine 3', 5'-monophosphate. PLoS ONE 4, e7649.

Munz, M., Gobert, D., Schohl, A., Poquérusse, J., Podgorski, K., Spratt, P., and Ruthazer, E.S. (2014). Rapid Hebbian axonal remodeling mediated by visual stimulation. Science 344, 904-909.

Nicol, X., and Gaspar, P. (2014). Routes to cAMP: shaping neuronal connectivity with distinct adenylate cyclases. Eur. J. Neurosci. 39, 1742-1751.

Nicol, X., Bennis, M., Ishikawa, Y., Chan, G.C.-K., Repérant, J., Storm, D.R., and Gaspar, P. (2006). Role of the calcium modulated cyclases in the development of the retinal projections. Eur. J. Neurosci. 24, 3401-3414.

Ohta, Y., Furuta, T., Nagai, T., and Horikawa, K. (2018). Red fluorescent cAMP indicator with increased affinity and expanded dynamic range. Sci. Rep. 8 , 1866.

Penn, A.A., Riquelme, P.A., Feller, M.B., and Shatz, C.J. (1998). Competition in retinogeniculate patterning driven by spontaneous activity. Science 279 , 2108-2112.

Pnevmatikakis, E.A., Soudry, D., Gao, Y., Machado, T.A., Merel, J., Pfau, D., Reardon, T., Mu, Y., Lacefield, C., Yang, W., et al. (2016). Simultaneous Denoising, Deconvolution, and Demixing of Calcium Imaging Data. Neuron 89, 285-299.

Ravary, A., Muzerelle, A., Hervé, D., Pascoli, V., Ba-Charvet, K.N., Girault, J.-A., Welker, E., and Gaspar, P. (2003). Adenylate cyclase 1 as a key actor in the refinement of retinal projection maps. J. Neurosci. 23, 2228-2238.

Ruthazer, E.S., Akerman, C.J., and Cline, H.T. (2003). Control of axon branch dynamics by correlated activity in vivo. Science 301, 66-70.

Shatz, C.J. (1983). The prenatal development of the cat's retinogeniculate pathway. J. Neurosci. 3, 482-499. 
Stellwagen, D., and Shatz, C.J. (2002). An instructive role for retinal waves in the development of retinogeniculate connectivity. Neuron 33, 357-367.

Stellwagen, D., Shatz, C.J., and Feller, M.B. (1999). Dynamics of retinal waves are controlled by cyclic AMP. Neuron 24, 673-685.

Stosiek, C., Garaschuk, O., Holthoff, K., and Konnerth, A. (2003). In vivo twophoton calcium imaging of neuronal networks. Proc. Natl. Acad. Sci. USA 100 7319-7324.
Yamamoto, N., and López-Bendito, G. (2012). Shaping brain connections through spontaneous neural activity. Eur. J. Neurosci. 35, 1595-1604.

Young, R.W. (1984). Cell death during differentiation of the retina in the mouse. J. Comp. Neurol. 229, 362-373.

Zhang, J., Ackman, J.B., Xu, H.-P., and Crair, M.C. (2011). Visual map development depends on the temporal pattern of binocular activity in mice. Nat. Neurosci. 15, 298-307. 


\section{STAR $\star$ METHODS}

\section{KEY RESOURCES TABLE}

\begin{tabular}{|c|c|c|}
\hline REAGENT or RESOURCE & SOURCE & IDENTIFIER \\
\hline \multicolumn{3}{|l|}{ Antibodies } \\
\hline rabbit anti-PKA1 $\beta$ antibody $(1 / 200)$ & Santa Cruz, Lot\# F2310 & Cat\# SC-907, RRID:AB_2237411 \\
\hline rabbit anti-RBPMS antibody $(1 / 500)$ & Phosphosolutions, lot\# NB317a & Cat\# 1830-RBPMS, RRID:AB_2492225 \\
\hline goat anti-rabbit HRP-coupled antibody (1/10000) & $\begin{array}{l}\text { Jackson ImmunoResearch, } \\
\text { Lot\# } 81283\end{array}$ & Cat\# 111035003 RRID:AB_2313567 \\
\hline donkey anti-rabbit Alexa Fluor-647 (1/500) & $\begin{array}{l}\text { Jackson ImmunoResearch, } \\
\text { lot\# } 129486\end{array}$ & Cat\# 711-605-152, RRID:AB_2492288 \\
\hline \multicolumn{3}{|l|}{ Biological Samples } \\
\hline Retinal explants & This manuscript & $\mathrm{N} / \mathrm{A}$ \\
\hline \multicolumn{3}{|l|}{ Chemicals, Peptides, and Recombinant Proteins } \\
\hline Ketamine & Axience & Cat\# Ketamidor $100 \mathrm{MG} / \mathrm{ML}$ \\
\hline Xylazine & Centravet & Cat\# Rompun $2 \%$ \\
\hline Buprenorphine & Axience & Cat\# Buprécare multidose 0,3MG/ML \\
\hline oxybuprocaine & CSP & $\begin{array}{l}\text { Cat\# Chlorhydrate d'oxybuprocaïne } \\
1,6 \mathrm{mg} / 0,4 \mathrm{ml}\end{array}$ \\
\hline Oregon Green BAPTA-1-AM & ThermoFisher & Cat\# 06807 \\
\hline Forskolin & Sigma-Aldrich & Cat\# F6886 \\
\hline cholera toxin subunit $\beta$ conjugated to AlexaFluor- 594 & ThermoFisher & Cat\# C34777 \\
\hline cholera toxin subunit $\beta$ conjugated to AlexaFluor- 647 & ThermoFisher & Cat\# C34778 \\
\hline DNase I recombinant, RNase-free & Roche & Cat\# 04716728001 \\
\hline \multicolumn{3}{|l|}{ Critical Commercial Assays } \\
\hline EndoFree Plasmid kits (maxi) & QIAGEN & Cat\# 12362 \\
\hline Lipofectamine 2000 & ThermoFisher & Cat\# 11668019 \\
\hline $\begin{array}{l}\text { Click-iT Plus TUNEL assay; Alexa Fluor-594 } \\
\text { connjugated }\end{array}$ & ThermoFisher & Cat\# C10618 \\
\hline \multicolumn{3}{|l|}{ Experimental Models: Cell Lines } \\
\hline HEK293T & ATCC & Cat\# CRL-1573. RRID: CVCL_0045 \\
\hline \multicolumn{3}{|l|}{ Experimental Models: Organisms/Strains } \\
\hline Mouse: C57BL/6NRj & Janvier Labs & RRID:MGI:6236253 \\
\hline Mouse: SWISS & Janvier Labs & RRID:MGI:2168141 \\
\hline \multicolumn{3}{|l|}{ Recombinant DNA } \\
\hline Plasmid: Sncg::Cre & This manuscript & $\mathrm{N} / \mathrm{A}$ \\
\hline Plasmid: CAG::Cre & Jean Livet laboratory & N/A \\
\hline Plasmid: SpongeZaic & This manuscript & $\mathrm{N} / \mathrm{A}$ \\
\hline Plasmid: ControlZaic & This manuscript & $\mathrm{N} / \mathrm{A}$ \\
\hline Plasmid: lox-STOP-lox-tdTomato & This manuscript & $\mathrm{N} / \mathrm{A}$ \\
\hline Plasmid: lox-STOP-lox-cAMP Sponge-2A-tdTomato & This manuscript & N/A \\
\hline Plasmid: RFlincA & Ohta et al., 2018 & $\mathrm{~N} / \mathrm{A}$ \\
\hline \multicolumn{3}{|l|}{ Software and Algorithms } \\
\hline MetaMorph & Molecular Devices & $\begin{array}{l}\text { https://www.moleculardevices.com/ } \\
\text { products/cellular-imaging-systems/ } \\
\text { acquisition-and-analysis-software/ } \\
\text { metamorph-microscopy }\end{array}$ \\
\hline Fiji & $\mathrm{NIH}$ & https://fiji.sc/ \\
\hline Plugin: Trainable Weka Segmentation & Arganda-Carreras et al., 2017 & https://imagej.net/Trainable_Segmentation \\
\hline MATLAB R2016a & MathWorks & https://www.mathworks.com/ \\
\hline
\end{tabular}

(Continued on next page) 


\begin{tabular}{|c|c|c|}
\hline \multicolumn{3}{|l|}{ Continued } \\
\hline REAGENT or RESOURCE & SOURCE & IDENTIFIER \\
\hline CalmAn & Pnevmatikakis et al., 2016 & $\begin{array}{l}\text { https://github.com/flatironinstitute/ } \\
\text { CalmAn-MATLAB }\end{array}$ \\
\hline GraphPad Prism & GraphPad & $\begin{array}{l}\text { https://www.graphpad.com/scientific- } \\
\text { software/prism/ }\end{array}$ \\
\hline R (v3.5.2) & The $R$ foundation & https://www.r-project.org/ \\
\hline Package: conover & R-project & $\begin{array}{l}\text { https://cran.r-project.org/web/packages/ } \\
\text { conover.test/index.html }\end{array}$ \\
\hline \multicolumn{3}{|l|}{ Other } \\
\hline \multirow[t]{2}{*}{ Electroporation paddles } & Sonidel & CUY650P5 \\
\hline & & CUY650P2 \\
\hline Electroporator & Nepagene & CUY21EDIT \\
\hline
\end{tabular}

\section{RESOURCE AVAILABILITY}

\section{Lead Contact}

Further information and requests for resources and reagents should be directed to and will be fulfilled by the Lead Contact, Xavier Nicol (xavier.nicol@inserm.fr).

Materials Availability

Requests for plasmids generated in this study should be directed to and will be fulfilled by the Lead Contact.

Data and Code Availability

This study did not generate any unique dataset or code that is central to supporting the main claims of the paper.

\section{EXPERIMENTAL MODEL AND SUBJECT DETAILS}

\section{Animals}

Timed-pregnant Swiss and C57BL/6NRj mice were purchased from Janvier Labs. All animal procedures were performed in accordance with institutional guidelines and approved by local ethics committees (C2EA-05: Comité d'éthique en expérimentation animale Charles Darwin). Animals were housed under 12h light / 12h dark cycle. Embryos from dated matings (developmental stage stated in each section describing individual experiments) were sexed at the end of some experiments (morphological analyses). The female to male ratio was close to 1 .

\section{Cell lines}

HEK293T cells (ATCC, not authenticated, free of mycoplasma contamination) were cultured in DMEM-Glutamax, supplemented with $10 \%$ fetal bovine serum (FBS) and $1 \%$ non-essential amino acids (NEAA, all from Invitrogen) and maintained in a $37^{\circ} \mathrm{C}, 5 \% \mathrm{CO} 2$ incubator.

\section{Retinal explants}

E14.5 embryos were isolated and kept in cold PBS. Embryos were decapitated and DNA was injected subretinally using an elongated borosilicate glass capillary (Harvard Apparatus). The following plasmids were used: ControlZaic $2 \mu \mathrm{g} \cdot \mu \mathrm{L}^{-1} ; \mathrm{CAG}:: \mathrm{Cre}_{0} \mu \mathrm{g} \cdot \mu \mathrm{L}^{-1}$, $0.1 \mu \mathrm{g} \cdot \mu \mathrm{L}^{-1}, 0.5 \mu \mathrm{g} \cdot \mu \mathrm{L}^{-1}, 2 \mu \mathrm{g} \cdot \mu \mathrm{L}^{-1}$. The success of DNA injection was assessed using $0.05 \%$ fast green supplemented to the DNA solution. The paddles of the electrodes (CUY650P5, Sonidel) were placed at the bottom and at the top of the head respectively. Two poring pulses (square wave, $175 \mathrm{~V}, 5 \mathrm{~ms}$ duration, with $50 \mathrm{~ms}$ interval) followed by four transfer pulses (40 V, $50 \mathrm{~ms}$ and $950 \mathrm{~ms}$ interpulse) were applied. The protocol was repeated with inverted polarities. After electroporation, the retinas were isolated and kept 24 hours in culture medium (DMEM-F12 supplemented with $1 \mathrm{mM}$ glutamine (Sigma Aldrich), 1\% penicillin/streptomycin (Sigma Aldrich), $0.01 \%$ BSA (Sigma Aldrich), $0.07 \%$ glucose), in a humidified incubator at $37^{\circ} \mathrm{C}$ and $5 \% \mathrm{CO}_{2}$.

\section{METHOD DETAILS}

\section{Plasmids}

All constructs used, except Sncg::Cre, were subcloned into a pCAG variant backbone and the Lyn-cAMP Sponge was previously described (Averaimo et al., 2016). The ControlZaic plasmid was obtained using a CFP variant mTurquoise2 flanked by two LoxP sites 
and followed by YFP. The SpongeZaic plasmid was obtained using a CFP variant mTurquoise2 flanked by two LoxP sites, attached to a Lyn-CAMP Sponge sequence fused to a YFP sequence by a self-cleaving 2A sequence (GGAAGCGGAGCTACTAACTT CAGCCTGCTGAAGCAGGCTGGAGACGTGGAGGAGAACCCTGGACCT). The SpongeZaic sequence was synthesized by Genscript and subloned into the PCAG vector. Lox-STOP-Lox Lyn-cAMP Sponge-2A-tdTomato was obtained by replacing the Turquoise2 sequence by its reverse complement and YFP by tdTomato. Further excision of the Lyn-cAMP Sponge sequence led to Lox-STOP-Lox-tdTomato. The CAG::Cre was a kind gift from Jean Livet. The Sncg promoter was provided by Deniz Dalkara (Chaffiol et al., 2017). To obtain the Sncg::Cre, the CAG promoter was replaced by the Sncg promoter followed by a multiple cloning site. Subsequently, the Cre sequence was inserted into the multiple cloning site.

\section{WESTERN-BLOT ANALYSIS}

HEK293T cells were transfected with various expression vectors using Lipofectamine 2000 according to the manufacturer's protocol (ThermoFisher). After 48h, total cellular proteins were extracted in lysis buffer (10 mM HEPES pH 7, 100 mM NaCl, 2 mM EDTA, 0.5\% NP-40) supplemented with protease inhibitor cocktail and phosphatase inhibitor cocktail 1 and 3 (Sigma-Aldrich). For immunoblotting, samples were separated on 4\%-15\% Mini-Protean TGX Tris-Glycine-buffer SDS-PAGE and transferred onto $0.2 \mu \mathrm{m}$ Trans-Blot Turbo nitrocellulose membranes (both Biorad). Membranes were blocked for $1 \mathrm{~h}$ at room temperature (RT) in 1X TBS (10 mM Tris $\mathrm{pH} 8.0,150 \mathrm{mM} \mathrm{NaCl}$,) supplemented with $5 \%(\mathrm{w} / \mathrm{v})$ dried skim milk powder. Primary antibody incubation was carried out overnight at $4{ }^{\circ}$ C, with rabbit anti-PKA1 $\beta$ antibody (SC-907; Santa Cruz; RRID:AB_2237411; 1/200). A goat anti-rabbit HRP coupled secondary antibody was used for detection (Jackson ImmunoResearch, West Grove, PA; 1/10000). In between and after antibody incubations, membranes were extensively washed in TBS-T (TBS containing 2.5\% Tween-20). Western blots were visualized using the enhanced chemiluminescence method (ECL prime Western Blotting detection reagent, Amersham).

\section{HEK293 cell transfection}

Lipocomplexes were formed using Lipofectamine 2000 in accordance with the manufacturer's instructions (ThermoFisher). The following plasmids were used for transfection: ControlZaic 100 ng; CAG::Cre 0 ng, 1 ng, 10 ng, 100 ng. Lipofectamine 2000 transfection reagent diluted in serum-free Opti-MEM media (ThermoFisher) was left at RT for 5 minutes, then added to the plasmids diluted in Opti-MEM at a lipid:DNA ratio of 2:1, mixed by gentle pipetting, and incubated at RT for 20 min. After forming the lipocomplexes, they were delivered to the media above the cells to deliver $0.15 \mu \mathrm{g} \cdot \mathrm{cm}^{-2}$ of DNA. The complexes remained in contact with the cells overnight. The cells were fixed in 4\% paraformaldehyde (PFA) 15 minutes at RT. The cells were finally washed in PBS and mounted in Mowiol (Sigma). Images were acquired under a 40X objective using an epifluorescence microscope (Leica DMI6000B).

\section{In utero retinal electroporation}

Timed-pregnant mice (Janvier Labs) were delivered to the animal facility a week prior to the surgery in order to allow a minimum of 5 days adaptation. In utero electroporation has been performed as previously described (Averaimo et al., 2016). In brief, C57BL/6NRj pregnant mice were anesthetized with an intraperitoneal injection of a Xylazine/Ketamine mix $\left(10 \mathrm{mg} \cdot \mathrm{kg}^{-1} \mathrm{and} 100 \mathrm{mg} \cdot \mathrm{kg}^{-1}, \mathrm{respec}-\right.$ tively) and a subcutaneous injection of buprenorphine $\left(0.0125 \mathrm{mg} \cdot \mathrm{kg}^{-1}\right)$ was made pre-surgery for analgesia. Midline laparotomy was performed, exposing uterine horns and allowing visualization of embryos. Left eye of E14.5 embryos was injected using an elongated glass capillary (Harvard apparatus) with different plasmid solutions. The success of DNA injection was assessed using $0.07 \%$ fast green supplemented to the DNA solution. The eye was then electroporated with 5 pulses of $45 \mathrm{~V}$ during $50 \mathrm{~ms}$ every $950 \mathrm{~ms}$ (Nepagene electroporator). In order to electroporate the ventro-temporal end of the retina, the negative electrode (CUY650P2, Sonidel) was positioned on the ventro-temporal part of the injected eye and the positive electrode on the opposite side of the head. To target the central part of the retina, the positive electrode (CUY650P5, Sonidel) was placed on the side of the injected eye. Following surgery, the incision site was sutured (4-0, Ethicon), and mice were allowed to give birth. To increase the survival of the electroporated pups, a Swiss adopting mouse was housed together with the mice that underwent surgery. The Swiss mouse, mated a day earlier than the C57BL/6NRj mice, gave birth one day earlier. At P0, only 2 Swiss pups were left in the cage so that the electroporated pups were adopted by the Swiss mouse.

\section{Confocal imaging}

P3 or P15 mice were deeply anesthetized with a mix of Xylazine/Ketamine $\left(20 \mathrm{mg} \cdot \mathrm{kg}^{-1}\right.$ and $200 \mathrm{mg} \cdot \mathrm{kg}^{-1}$, respectively), perfused transcardially with 4\% PFA in $0.12 \mathrm{M}$ phosphate buffer. Retinas, optic chiasms and brains were dissected out and postfixed in $4 \%$ PFA. Retinas (oriented with an incision on the ventral part) and chiasms were mounted in Mowiol. Brains were cryoprotected with $30 \%$ sucrose in PB during 2 days and sectioned at $80 \mu \mathrm{m}$ thickness on a freezing microtome (Thermoscientific HM450). Brains sections were mounted in Mowiol. To validate the area of electroporation, retinas were imaged under a 2.5X objective using an epifluorescence microscope (Leica DMI6000B). In order to quantify the number of electroporated axons, optic chiasms were imaged using a confocal microscope (Olympus FV1000; 20X objective, N.A. 0.85). Electroporated brain sections were imaged under a confocal microscope (Olympus FV1000; 40X objective, N.A. 1.3). 
Plasmids electroporated

The plasmids used are summarized in Table S2.

Retina slicing and immunostaining

Electroporated eyes of P3 mice were dissected out, immersed overnight in 4\% PFA in PB, then cryoprotected in gelatin-sucrose. Eyes were frozen at $-40^{\circ} \mathrm{C}$ in propanol, then sectioned at $20 \mu \mathrm{m}$ using a cryostat (Leica CM3050S). Slices were permeabilized and blocked with PBS gelatin-sucrose (PBSGT), then incubated with antibodies against RBPMS (RNA-binding protein with multiple splicing, 1830-RBPMS, Phosphosolutions, lot \#NB317a; RRID:AB_2492225; 1/500) followed by a secondary antibody coupled to AlexaFluor-647 (711-605-152, Jackson, lot \#129486; RRID:AB_2492288; 1/500).

\section{Two-photon calcium imaging}

For the calcium imaging experiment the central retina was targeted during in utero electroporation. Mice were taken at postnatal day 7-8 (P7-8). The pup was rapidly decapitated, the left eye removed and placed in Ringer's medium containing (in $\mathrm{mM}$ ): $119 \mathrm{NaCl}$, $2.5 \mathrm{KCl}, 1.0 \mathrm{KH}_{2} \mathrm{PO}_{4}, 11$ glucose, $26.2 \mathrm{NaHCO}_{3}, 2 \mathrm{CaCl}_{2}$ and $1 \mathrm{MgCl}_{2}$ (290-295 mOsm), bubbled with carbogen $\left(95 \% \mathrm{O}_{2} / 5 \%\right.$ $\mathrm{CO}_{2}$ ). The retina was carefully dissected and fixed on filter paper (N8895, Sigma-Aldrich) with the RGC layer upward. The chamber containing the retina was placed in custom-made two-photon setup where it was perfused continuously with Ringer's solution. Widefield fluorescence was observed to select a suitable area with a high number of fluorescent RGCs. This area was loaded for $1 \mathrm{~h}$ at RT with Oregon Green BAPTA-1-AM (OGB1, 1 mM, O6807, ThermoFisher) following the multi-cell bolus loading technique (Stosiek et al., 2003). Calcium waves were imaged at $32-34^{\circ} \mathrm{C}$. A pulsed femtosecond laser (InSight DeepSee; Newport Corporation) was set to $920 \mathrm{~nm}$ for OGB1 and to $1050 \mathrm{~nm}$ for tdTomato. Imaging was performed with a 25X objective (XLPLN25xWMP/NA1.05, Olympus) and captured by a PMT (Hamamatsu, DP-Type Socket assembly, C12597-01). To suppress tdTomato fluorescence during calcium imaging we used a Chroma filter (ET535/50) placed before the PMT. The field of view was $0.5 \times 0.5$ or $0.25 \times 0.25 \mathrm{~mm}$ with a lateral resolution of 0.75 or $1 \mathrm{pixel} / \mu \mathrm{m}$. Acquisition rates ranged from $0.9 \mathrm{~Hz}$ to $3.6 \mathrm{~Hz}$. Multiple $15-30$ minutes movies were captured. After each movie the retina was allowed to recover for 30 minutes.

\section{Whole-cell electrophysiology}

The retina was isolated as described for the two-photon calcium imaging experiments and left to recover for at least $1 \mathrm{~h}$. Widefield RGC fluorescence was checked using a Leica EL6000, Leica filter cubes L5 for GFP, N3 for tdTomato, and Chroma EYFP filter cube (49003), and a Hamamatsu ORCA-ER camera. An area with a high number of fluorescent RGCs was selected. Thick-walled borosilicate pipettes (OD/ID of 1.5/0.87 mm; 30-0060, Harvard Apparatus) were pulled on a P-1000 Flaming/Brown puller (Sutter Instruments). Pipettes were filled with intracellular solution containing (in mM): $128 \mathrm{~K}$-gluconate, $10 \mathrm{HEPES}, 16 \mathrm{KCl}, 1 \mathrm{EGTA}, 2 \mathrm{Mg}-\mathrm{ATP}$, $0.5 \mathrm{Na}_{2}$-GTP, pH 7.25 with $\mathrm{KOH}$ (275 mOsm). In some recordings 10-20 $\mu$ M Alexa Fluor 488 or 594 hydrazide (A10436, A10438, ThermoFisher) and/or $2 \mathrm{mg} / \mathrm{mL}$ biocytin (B4261, Sigma-Aldrich) was added to the pipette solution to validate the recorded cells. Reported potentials were corrected for a liquid junction potential of $-11 \mathrm{mV}$. A pipette with tip resistance of 3-6 $\mathrm{M} \Omega$ was gently pushed through the inner limiting membrane with the pipette solution pressurized to keep the tip clean. Fluorescent cells and surrounding non-fluorescent cells in the RGC layer were targeted. Whole-cell recordings were made with a Multiclamp 700B amplifier (Axon Instruments), filtered at $10 \mathrm{kHz}$ and digitized at 25 or $50 \mathrm{kHz}$ using a DigiData 1440A (Axon Instruments) and Clampex 10.7 running on Windows 10. High-resistance patch seals $(>1 \mathrm{G} \Omega$ ) were obtained before breaking into the cell. Recordings with a series resistance above $50 \mathrm{M} \Omega$ were discarded. The resting membrane potential of the cell was recorded in the first minute after breaking in. Cells with a membrane potential $>-45 \mathrm{mV}$ were discarded. Passive cell properties were recorded by stepping from -70 to $-80 \mathrm{mV}$ in voltage-clamp mode. In current-clamp mode series resistance (10-50 M $\Omega$ ) and pipette capacitance (6.2-7.1 pF) were fully compensated. RGC responses to square current injections were recorded. The amplitude of first current injection was adjusted to hyperpolarize the cell to $-100 \mathrm{mV}$. Steps of current were then injected until reaching the action potential (AP) threshold. Cells that did not fire APs were discarded. Lastly, 15-30 minutes of spontaneous activity were recorded. During the recording, fluorescence was checked to categorize the cell as positive or negative for fluorescence of YFP or tdTomato, depending on the plasmid construct that was electroporated.

\section{TUNEL staining and analysis}

P3 wild-type or electroporated retinas were dissected out and postfixed $1 \mathrm{~h}$ with 4\% PFA in PB. Developmental cell death was assessed on whole retinas using TUNEL staining, in accordance with the manufacturer's instructions (ThermoFisher, C10618). To induce DNA strands breaks (i.e., positive control), retinas were incubated with 1 unit of DNase I (04716728001, Roche) for 30 min at room temperature. Slices were mounted in Mowiol then imaged using a confocal microscope (Olympus FV1000; 40X objective, N.A. 1.3) and the number of TUNEL-positive cells was manually counted.

\section{R-FlincA imaging}

Retinal explants were electroporated with the R-FlincA, CAG::Cre and SpongeZaic or ControlZaic constructs. The day after electroporation, they were cut into $0.2 \times 0.2 \mathrm{~mm}$ squares with a Tissue-Chopper (Mcllwan) and explants were plated on glass coverslips coated with poly-L-lysine and laminin (Sigma Aldrich). Cells were cultured for 24 hours in culture medium supplemented with $0.4 \%$ methylcellulose and B-27 (Life Technologies) before imaging. Images were acquired every $20 \mathrm{~s}$ with an inverted DMI6000B 
epifluorescence microscope (Leica) coupled to a 40X oil-immersion objective (N.A. 1.3), a TX2 filter cube and the Metamorph software (Molecular Devices). For live imaging experiments, cells co-transfected with R-FlincA and SpongeZaic or ControlZaic were perfused $\left(0.3 \mathrm{~mL} \mathrm{~min}^{-1}\right.$ ) with $1 \mathrm{mM} \mathrm{CaCl}, 0.3 \mathrm{mM} \mathrm{MgCl}_{2}, 0.5 \mathrm{mM} \mathrm{Na}_{2} \mathrm{HPO}_{4}, 0.45 \mathrm{mM} \mathrm{NaH}_{2} \mathrm{PO}_{4}, 0.4 \mathrm{mM} \mathrm{MgSO}_{4}, 4.25 \mathrm{mM} \mathrm{KCl}_{\text {, }}$ $14 \mathrm{mM} \mathrm{NaHCO}, 120 \mathrm{mM} \mathrm{NaCl}, 0.0004 \% \mathrm{CuSO}_{4}, 0.124 \mu \mathrm{M} \mathrm{Fe}\left(\mathrm{NO}_{3}\right)_{3}, 1.5 \mu \mathrm{M} \mathrm{FeSO}, 1.5 \mu \mathrm{M}$ thymidine, $0.51 \mu \mathrm{M}$ lipoic acid, $1.5 \mu \mathrm{M} \mathrm{ZnSO}_{4}, 0.5 \mathrm{mM}$ sodium pyruvate (all from Sigma), 1X MEM Amino Acids (Life Technologies), 1X non-essential amino acids (Life Technologies), 25 mM HEPES (Sigma), $0.5 \mu$ M putrescine (Sigma), 0.01\% BSA (Sigma), 0.46\% glucose (Sigma), 1 mM glutamine (Life Technologies), 2\% penicillin streptomycin (Life Technologies). Vitamin B12 and riboflavin were omitted because of their autofluorescence. Forskolin (Sigma) was used at $10 \mathrm{nM}$.

\section{Anterograde tracing}

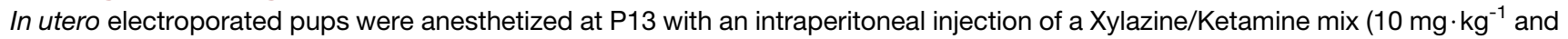
$100 \mathrm{mg} \cdot \mathrm{kg}^{-1}$, respectively), and oxybuprocaine $\left(4 \mathrm{mg} \cdot \mathrm{mL}^{-1}\right)$ was used for local analgesia. They were injected with a Nanofil syringe intravitreally with $2 \mu \mathrm{L}$ of cholera toxin subunit $\beta$ (ThermoFisher) conjugated to AlexaFluor-647 (left eye) or 594 (right eye) diluted in $1 \%$ DMSO. P15 mice were perfused transcardially with 4\% PFA in $0.12 \mathrm{M}$ phosphate buffer. Brain sections were imaged under a confocal microscope (Olympus FV1000; 10X objective, N.A. 0.4).

\section{QUANTIFICATION AND STATISTICAL ANALYSIS}

\section{Axon area analysis}

Axon density analysis was performed on 40X confocal images from all the coronal sections containing the dLGN contralateral to the electroporated eye. Z stack images of axons were segmented using a machine-learning based plugin of Fiji: Trainable Weka Segmentation plugin (Arganda-Carreras et al., 2017). Briefly the algorithm was manually trained for several rounds with a set of images using the default settings of the Weka plugin. The robustness of the training was confirmed on a different set of images. Once stabilized, the segmentation process was applied to all quantified images. After segmentation, axons expressing both YFP and CFP were excluded from the CFP segmented image, in order to obtain a 'CFPOnly' stack of images (Figures S1J and S1K). A region of interest (ROI) was manually drawn around the patch of axons, and the area covered by axons inside this ROI was measured on the maximal projection of the segmented stacks of YFP and 'CFPOnly' axons. Axon area was summed on all the coronal slices of the dLGN.

\section{Analysis of the distribution of ipsilateral and contralateral fibers in the dLGN}

Quantifications were performed on 10X confocal images from the 3 consecutive coronal sections containing the largest extent of the ipsilateral territory. Using ImageJ software, the boundary of the dLGN was outlined, excluding the intrageniculate leaflet, the vLGN and the optic tract. The proportion of ipsilateral projections within the dLGN was measured as a ratio of ipsilateral pixels to the total number of pixels in the dLGN. The area of ipsilateral projections was summed over the 3 largest consecutive coronal sections of the dLGN.

\section{Analysis of maximal invading distance}

The maximal distance to which axons invade the dLGN of P3 electroporated mice was manually measured on 40X confocal images. Axon length was measured on the five longest axons on three consecutive coronal sections containing the greatest quantity of retinal axons.

\section{Calcium imaging analysis}

The OGB fluorescence movie $Y_{i, j, t}$ and a tdTomato fluorescence image were imported into MATLAB R2016a (MathWorks). The tdTomato image was visually aligned to $Y$ (rigid transformation).

\section{Defining ROls}

Centers of ROIs were defined by the user by viewing the overlay of the tdTomato image and the standard deviation time projection of $\mathrm{Y}_{\mathrm{i}, \mathrm{j}, \mathrm{t}}$. Pixels within $4 \mu \mathrm{m}$ from the center were included in the initial ROI unless these pixels were already assigned to another ROI. In a few cases when variations in OGB signals were not sufficient to define the center of the cell, ROls were defined based on the tdTomato image. A threshold value was defined by the median $+0.2^{*}$ (maximum - median) of the tdTomato image after a $2 \mathrm{D}$ Gaussian smoothing kernel $(S D=5 \mu \mathrm{m})$. Any pixels above this threshold connected to the defined center would be part of the initial ROI.

Subsequently, the spatial and temporal components were initialized following the Greedy Initialization as described by Pnevmatikakis et al., 2016. In brief, $Y_{i, j, t}$ was Gaussian-blurred (2D, SD = $\left.2 \mu \mathrm{m}\right)$, subtracted by a pixel-based median, and averaged over the $\mathrm{ROI}$ pixels to obtain the initial temporal component. This was followed by the fine-tuning step of Greedy Initialization to adjust the spatial and temporal components of the ROI. Pixels were excluded when their spatial component value was below $0.7^{\star}$ median intensity of all spatial component values. Finally, spatial and temporal components of the ROls were updated following Pnevmatikakis et al., 2016. Any $\mathrm{ROI}<12.5 \mu \mathrm{m}^{2}$ or $<16$ pixels was excluded. 
ROIs were classified as either tdTomato positive or negative as follows. Mean intensity of tdTomato fluorescence was calculated for each ROI. Based on the average intensity and the SD of ROls below the $80^{\text {th }}$ percentile an intensity threshold was calculated as mean intensity $+6^{*} \mathrm{SD}$. ROls with at least $70 \%$ of its pixels and an average tdTomato intensity above this threshold were identified as tdTomato-positive ROls.

\section{Activity of the ROls}

By multiplying the spatial and temporal component of a ROI we obtained its background-subtracted fluorescence trace. From this trace we calculated $\Delta \mathrm{F} / \mathrm{F}$ by $\left(\mathrm{Y}_{\mathrm{t}}-\mathrm{Y}_{\text {median }}\right) / \mathrm{Y}_{\text {median, }}$, and $\Delta \mathrm{F} / \mathrm{F}$ was detrended by subtracting a 3 minutes moving median. At first, we defined the 'active' time points when $\Delta F / F>0.2$. However, this causes ROls with low median intensities to cross the threshold very often. To avoid this, we made the threshold dependent on the $Y_{i, j, t}$ of the ROI. The 3 minutes moving median of $Y_{R O I, t}\left(Y_{m e d} m_{R O, t}\right)$ was calculated. The activity threshold was calculated as $\max \left(0.2,0.4-\mathrm{Ymed}_{\mathrm{RO}, \mathrm{t}} / 1250\right)$ and ROIs were considered active when both $\Delta \mathrm{F} /$ $\mathrm{F}>$ the activity threshold and $\mathrm{Ymed}_{\mathrm{ROI}, \mathrm{t}}>20$. ROls that had an active period $>30 \mathrm{~s}$ were excluded from the analysis.

\section{Defining waves}

Waves were detected when $>5 \%$ of the ROls that are active in the movie were active simultaneously. The start and end of a wave was defined as the time point that $4 \%$ threshold was reached before and after crossing the $5 \%$ threshold. To define the wave area, a convex hull was drawn for every time point around active ROls within the wave with a centroid-centroid distance $<50 \mu \mathrm{m}$. The final wave area was the total area covered by the individual hulls. To calculate the participation, the number of active ROls within the wave area was divided by the total number of ROls with at least 1 pixel in the wave area.

\section{Electrophysiology analysis}

Recordings were imported into MATLAB R2016a. Series and input resistance were calculated from the $10 \mathrm{mV}$ voltage steps. The membrane resistance was calculated as the difference of the two resistances. For the current injection responses, we analyzed the AP properties of the first AP at the beginning of each current injection step. AP threshold was defined as the membrane potential at which $200 \mathrm{kV} \cdot \mathrm{s}^{-2}$ was reached. Maximal rate of rise and maximal rate of fall were taken as the maximum and minimum in the first derivative of the AP. For a few cells the AP maximal rate of rise corresponds to axon initial segment spike as judged from the phase plot. Full-width at half-maximum (FWHM) was taken as the duration where the AP was above the membrane potential of the AP threshold + 0.5 (AP peak - AP threshold). In the spontaneous activity APs were detected in the 15-30 minutes recordings by threshold crossing at $-25 \mathrm{mV}$. Bursts were defined as $>2$ AP within $0.5 \mathrm{~s}$. Cells with less than four bursts were discarded.

\section{R-FlincA imaging analysis}

Images were imported into MATLAB R2016A. ROls were drawn by the experimenter on axons that (1) were structurally stable throughout the experiment and (2) showed R-FlincA fluorescence above background levels ( $>0.5$ arbitrary points). For each ROI a background $\mathrm{ROI}$ was drawn next to it. Average fluorescence in ROI was subtracted by the average fluorescence in the background $\mathrm{ROI}$ at each time point $\left(F_{\mathrm{t}}\right)$. Baseline fluorescence was calculated by averaging the first 15 frames $\left(F_{0}\right)$. The average of $F / F_{0}$ in $1^{\text {st }}-10^{\text {th }}$ frame after the start of the forskolin pulse was used to measure the R-FlincA response. YFP fluorescence was calculated by averaging the pixels within the ROI and subtracting the average from the background ROI. In some cases the forskolin pulse induced a shift in $\mathrm{Z}$ in the following image with the structures being out-of-focus. This image was then excluded from the averages.

\section{Statistics}

No data were excluded from the analysis, except where indicated otherwise. No sample size calculation was performed. Non-parametric (Mann-Whitney and Kruskal-Wallis) tests were calculated using GraphPad Prism (GraphPad software Inc.) or using package conover in R (v3.5.2). Post hoc comparisons were done following the Conover-Iman test using package conover. When the KruskalWallis test indicated a p value $<0.05$, post hoc contrasts were made between (1) CAMP $^{\text {reduced }}$ cells versus Control $\mathrm{YFP}^{+}$or tdTom $^{+}$, and (2) CAMP ${ }^{\text {intact }}$ versus Control YFP' or tdTom ${ }^{-}$. These contrasts were corrected following Bonferroni and indicated as $\mathrm{p}^{\prime}$. When $\mathrm{p}^{\prime}$ was $>1$, $p$ was reported instead. When none of the predefined contrasts reached $p^{\prime}<0.10$, we explored the comparisons Control $\mathrm{YFP}^{+}$or tdTom ${ }^{+}$versus Control nearby and cAMPreduced versus cAMP ${ }^{\text {intact }}$. These comparisons are reported without any correction. R-FlincA experiments were analyzed using a regression model to explain the ranks of R-FlincA response by the ranks of YFP intensity, a logical variable for ControlZaic and SpongeZaic, and the interaction effect. Post hoc comparison tests were corrected following the Bonferroni correction. Table S1 summarizes the $\mathrm{p}$ values and number of replicates for all the data. 